\title{
Sensitivity of lepton number violating meson decays in different experiments
}

\author{
Eung Jin Chun, ${ }^{1, *}$ Arindam Das, ${ }^{2, \dagger}$ Sanjoy Mandal@ ${ }^{3,4, \sharp}$ Manimala Mitra, ${ }^{3,4, \S}$ and Nita Sinha ${ }^{5,4, \|}$ \\ ${ }^{1}$ Korea Institute for Advanced Study, Seoul 130-722, Korea \\ ${ }^{2}$ Department of Physics, Osaka University, Toyonaka, Osaka 560-0043, Japan \\ ${ }^{3}$ Institute of Physics, Sachivalaya Marg, Bhubaneswar 751005, India \\ ${ }^{4}$ Homi Bhabha National Institute, BARC Training School Complex, Anushakti Nagar, \\ Mumbai 400094, India \\ ${ }^{5}$ The Institute of Mathematical Sciences, C.I.T. Campus, Taramani, Chennai 600 113, India
}

(Received 5 September 2019; published 20 November 2019)

\begin{abstract}
We study the discovery prospect of different three body lepton number violating (LNV) meson decays $M_{1}^{-} \rightarrow \ell_{1}^{-} \ell_{2}^{-} M_{2}^{+}$in the framework of the right-handed (RH) neutrino extended Standard Model. We consider a number of ongoing experiments, such as NA62 and LHCb at CERN, Belle II at SuperKEK, as well as at the proposed future experiments, SHiP, MATHUSLA and FCC-ee. The RH Majorana neutrino $N$ mediating these meson decays provides a resonant enhancement of the rates, if the mass of $N$ lies in the range (100 MeV-6 GeV). We consider the effect of parent mesons velocity, as well as the effect of finite detector size. Using the expected upper limits on the number of events for the LNV decay modes, $M_{1}^{-} \rightarrow$ $\ell_{1}^{-} \ell_{2}^{-} \pi^{+}\left(M_{1}=B, B_{c}, D, D_{s}\right.$ and $\left.K\right)$, we analyze the sensitivity reach of the mixing angles $\left|V_{e N}\right|^{2},\left|V_{\mu N}\right|^{2}$, $\left|V_{\tau N}\right|^{2},\left|V_{e N} V_{\mu N}\right|,\left|V_{e N} V_{\tau N}\right|$ and $\left|V_{\mu N} V_{\tau N}\right|$ as a function of heavy neutrino mass $M_{N}$. We show that inclusion of parent meson velocity can account to a large difference for active-sterile mixing, especially for $D, D_{s}$ meson decay at SHiP and $K$ meson decay at NA62. Taking into account the velocity of the $D_{s}$ meson, the future beam dump experiment SHiP can probe $\left|V_{e N}\right|^{2} \sim 10^{-9}$. For RH neutrino mass in between 2-5 GeV, MATHUSLA can provide the best sensitivity reach of active-sterile mixings.
\end{abstract}

DOI: $10.1103 /$ PhysRevD.100.095022

\section{INTRODUCTION}

The discovery of neutrino oscillations [1] in a series of oscillation experiments has confirmed that neutrinos have nonzero masses and nonzero mixings. The solar and atmospheric mass splittings are $\mathcal{O}\left(10^{-5}\right)$ and $\mathcal{O}\left(10^{-3}\right) \mathrm{eV}^{2}$, while the three mixings are $\theta_{12} \sim 33^{\circ}$, $\theta_{23} \sim 45^{\circ}$ and $\theta_{13} \sim 9^{\circ}$. These observations indicate at least two of the three Standard Model (SM) neutrinos have nonzero masses. The absolute scale of the neutrino masses is yet unknown. The sum of masses of three active neutrinos is bounded from cosmological observation as $\sum_{i} m_{\nu_{i}}<0.23 \mathrm{eV}[2]$. One of the most natural Ansätze to explain small neutrino masses is the seesaw mechanism, where the dimension-5 operator [3] with lepton and Higgs

\footnotetext{
*ejchun@kias.re.kr

tarindam.das@het.phys.sci.osaka-u.ac.jp

smandal@iopb.res.in

\$manimala@iopb.res.in

"nita@imsc.res.in
}

Published by the American Physical Society under the terms of the Creative Commons Attribution 4.0 International license. Further distribution of this work must maintain attribution to the author(s) and the published article's title, journal citation, and DOI. Funded by SCOAP ${ }^{3}$. doublets generates Majorana mass of light neutrinos through electroweak symmetry breaking. This operator breaks global lepton number symmetry of the SM by two units. The other possibility is to generate Dirac mass terms for the SM neutrinos by including gauge singlet right-handed $(\mathrm{RH})$ neutrinos in the theory. However, to explain $\mathrm{eV}$ mass of the neutrinos, this requires unnatural fine-tuning of Yukawa coupling to a very small value $Y_{\nu} \sim \mathcal{O}\left(10^{-11}\right)$. The seesaw mechanism on the other hand is most appealing, as the tiny Majorana mass of the light neutrinos is inversely proportional to the cutoff scale of the theory. This large cutoff scale naturally explains $\mathrm{eV}$ mass of neutrinos. Seesaw can be realized in different beyond standard model extensions, such as type-I [4-7]/inverse seesaw [8,9] with gauge singlet Majorana neutrinos, type-II [10-13] seesaw with Higgs triplets, and type-III seesaw [14-17] with fermionic triplet. For type-I/inverse seesaw, RH neutrinos can have Majorana/quasi-Dirac masses, which can vary in wide ranges, starting from grand unified theory scale down to GeV. The low scale seesaw models that inherit lighter RH neutrino states have higher discovery prospect, as they can be tested in a wide range of experiments.

Heavy SM gauge singlet RH neutrinos of mass $\mathrm{GeV}$ to $\mathrm{TeV}$ can be searched at LHC, via dilepton [18-23], as well 
as trilepton final states [24-29]. The $13 \mathrm{TeV}$ LHC trilepton search has constrained the mixing of the RH neutrinos with active neutrinos down to $\left|V_{\ell N}\right|^{2}<10^{-5}$ [28], for the mass of RH neutrino $M_{N}$ in between 10 to $50 \mathrm{GeV}$. The displaced vertex signature can also give a very tight constraint on mixing angles $\left|V_{\ell N}\right|^{2} \sim 10^{-9}$ for right-handed neutrinos at the high luminosity LHC for mass range between $5 \mathrm{GeV}<$ $M_{N}<30 \mathrm{GeV}$ [30]. For other future colliders, such as $e^{+} e^{-}$, FCC-hh, and discussions on a future $e^{-} p$ collider, such as LHeC, see [31-38]. For the heavy RH neutrino of mass $M_{N} \sim \mathrm{TeV}$, the final decay products will be collimated and will produce fat jets [23,34,36,39]. For the discussion on sub-weak-scale RH neutrino state that can produce lepton jet, see [40]. The other mass ranges of RH neutrinos, such as $\mathrm{MeV}-\mathrm{GeV}$, are tightly constrained from the lepton number violating neutrinoless double beta decay

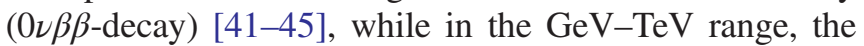
lepton flavor violating process $\mu \rightarrow e \gamma$ [46-48] can give significant constraint. RH neutrinos have also been searched for in the laboratory experiment through peak searches in leptonic decays of pions and kaons [49,50].

Another interesting probe for Majorana neutrinos of hundreds of $\mathrm{MeV}$-few $\mathrm{GeV}$ masses and their mixings are the lepton number violating (LNV) rare meson decay processes, such as $M_{1}^{-} \rightarrow \ell_{1}^{-} \ell_{2}^{-} \pi^{+}$[50-66]. For the RH neutrino search, this process has an advantage as compared to the LNV $0 \nu \beta \beta$-decay due to the lesser uncertainties in the meson decay constant. In the later process, the nuclear matrix elements uncertainty can make a difference in the prediction of active-sterile mixing. We consider a number of three body $\Delta L=2$ meson decays $M_{1}^{-} \rightarrow \ell_{1}^{-} \ell_{2}^{-} \pi^{+}$ $\left(M_{1}=B, B_{c}, D, D_{s}\right.$ and $\left.K\right)$ and derive the sensitivity reach of the active-sterile mixing parameter in a number of ongoing and future experiments, such as NA62, LHCb at CERN, Belle II, SHiP, MATHUSLA and FCC-ee. Note that the light neutrino contribution to these meson decays is extremely suppressed. However, for RH neutrinos in the mass range of $0.140 \mathrm{GeV}<M_{N}<6 \mathrm{GeV}$, the intermediate Majorana neutrinos can be produced on shell. This results in resonant enhancement of these decay rates. In addition, a large number of decaying mesons in these experiments, in particular at $\mathrm{SHiP}$, will facilitate to improve the sensitivity reach significantly. In deriving these results, we consider parent meson velocity that affects the probability of RH neutrino decay inside the detector. We show that inclusion of parent meson velocity can give 1 or 2 orders of magnitude shift $\mathcal{O}\left(10^{1}-10^{2}\right)$ in the results obtained.

The paper is organized as follows. In Sec. II, we very briefly review the basic features of the three RH neutrino framework, following which in Sec. III we discuss in detail the contributions of the RH neutrino in meson decays. In Sec. IV, we then compute the total decay width of RH neutrino $N$ in the mass range $0.140 \mathrm{GeV} \leq M_{N} \leq 6 \mathrm{GeV}$. In Sec. V, we study the effects of parent meson velocity in the RH neutrino decay probability. In Secs. VI and VII, we give the formalism to calculate the signal events and give different inputs for various experiments which we consider. In Sec. VIII, we derive the limits on the mixing angle $\left|V_{\ell N}\right|^{2}, \ell=e, \mu, \tau$ and $\left|V_{\ell_{1} N} V_{\ell_{2} N}\right|, \ell_{1}, \ell_{2}=e, \mu, \tau, \ell_{1} \neq$ $\ell_{2}$ that are expected from the upper limits on the number of signal events of various LNV meson decays that may be achievable in some of the ongoing and future experiments. In Sec. IX, we present our combined limit from all the considered meson decays and give the comparison with other existing constraints on the mixing angles. Finally in Sec. X, we provide our conclusions. In the Appendix, we provide details of the RH neutrino decay width calculations.

\section{THE MODEL}

We extend the SM to include additional RH neutrinos $N$. The heavy neutrinos can generate light neutrino masses through seesaw. For simplicity, we consider only one RH neutrino and carry out our analysis. The mixing of $N$ with the active neutrinos is given by the following expression:

$$
\nu_{\ell}=\sum_{m=1}^{3} U_{\ell m} \nu_{m}+V_{\ell N} N_{m^{\prime}}^{c},
$$

where $\nu_{m}$ and $N_{m^{\prime}}$ are the mass eigenstates. We denote the mixing between the standard flavour neutrino $\nu_{\ell}$ $(\ell=e, \mu, \tau)$ and the heavy mass eigenstate $N$ by $V_{\ell N}$. Due to the mixing, the charged and neutral currents in the lepton sector gets modified and can be written as

$$
\begin{aligned}
\mathcal{L}_{\ell}^{C C}= & -\frac{g}{\sqrt{2}} W_{\mu}^{+}\left(\sum_{\ell=e}^{\tau} \sum_{m=1}^{3} U_{\ell m}^{*} \bar{\nu}_{m} \gamma^{\mu} P_{L} \ell\right. \\
& \left.+\sum_{\ell=e}^{\tau} V_{\ell N}^{*} \overline{N_{m^{\prime}}^{c}} \gamma^{\mu} P_{L} \ell\right)+ \text { H.c., } \\
\mathcal{L}_{\ell}^{N C}=- & \frac{g}{2 \cos \theta_{W}} Z_{\mu}\left(\sum_{\ell=e}^{\tau} \sum_{m=1}^{3} U_{\ell m}^{*} \bar{\nu}_{m} \gamma^{\mu} P_{L} \nu_{\ell}\right. \\
& \left.+\sum_{\ell=e}^{\tau} V_{\ell N}^{*} \overline{N_{m^{\prime}}^{c} \gamma^{\mu}} P_{L} \nu_{\ell}\right)+ \text { H.c. }
\end{aligned}
$$

For the purpose of phenomenology, we consider the mass and mixings of $N$ as free parameters, constrained only by experimental observations. Note that adding only one RH neutrino is not enough to correctly reproduce the neutrino oscillations parameters, namely two mass square differences and the mixings. In our considered model, we can add two more $\mathrm{RH}$ neutrinos to generate the neutrino masses and consider two of them to be heavy enough such that only one RH neutrino lies in the mass range $0.140 \mathrm{GeV} \leq M_{N} \leq 6 \mathrm{GeV}$.

\section{PROCESS}

The RH neutrino $N$, if a Majorana state can mediate the LNV process $M_{1}^{-} \rightarrow \ell_{1}^{-} \ell_{2}^{-} M_{2}^{+}$. The Feynman diagrams for 

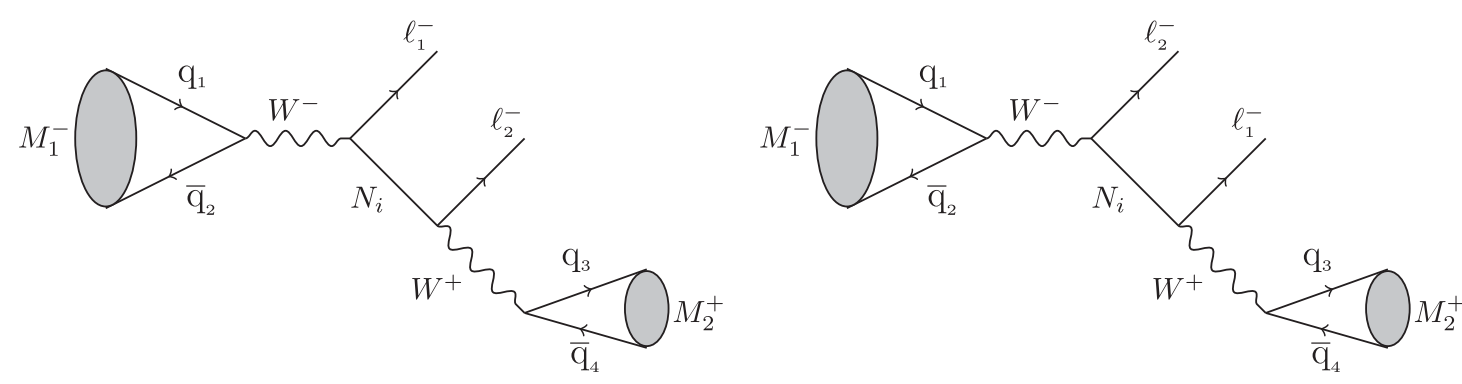

FIG. 1. The Feynman diagrams for the lepton number violating meson decays. These processes can produce resonance enhancement. See the text for details.

these decays are shown in Figs. 1 and 2. The diagram in Fig. 2 will give a very small contribution, as this is not a resonance production diagram. Note that the diagrams with light neutrino exchange are also present but the contributions will be negligibly small as they will not be resonantly enhanced. The decay amplitude for the processes $M_{1}^{-}(p) \rightarrow$ $\ell_{1}^{-}\left(k_{1}\right) \ell_{2}^{-}\left(k_{2}\right) M_{2}^{+}\left(k_{3}\right)$ depicted in Fig. 1 can be expressed as

$$
\begin{aligned}
\mathcal{M}= & G_{F}^{2} V_{M_{1}}^{\mathrm{CKM}} V_{M_{2}}^{\mathrm{CKM}} f_{M_{1}} f_{M_{2}}\left(V_{\ell_{1} N} V_{\ell_{2} N}\right) \\
& \times \frac{M_{N}}{\left(p-k_{1}\right)^{2}-M_{N}^{2}+i M_{N} \Gamma_{N}} \bar{u}\left(k_{1}\right) \not p k_{3}\left(1+\gamma_{5}\right) v\left(k_{2}\right),
\end{aligned}
$$

where $M_{1}$ and $M_{2}$ are both pseudoscalar mesons. Though $M_{2}$ can also be a vector meson, we have considered only the case of pseudoscalar meson. In Eq. (4), $G_{F}$ is the Fermi coupling constant, $V_{\ell_{j} N}$ are the mixing angles between the neutrino of flavor state $\nu_{\ell_{j}}$ and mass eigenstate N. $V_{M_{1}}^{\mathrm{CKM}}\left(V_{M_{2}}^{\mathrm{CKM}}\right)$ are the Cabbibo-Kobayashi-Maskawa (CKM) matrix elements at the annihilation (creation) vertex of the meson $M_{1}\left(M_{2}\right) . f_{M_{1}}$ and $f_{M_{2}}$ are the decay constants of $M_{1}$ and $M_{2}$, respectively. We use the values $f_{D}=0.204 \mathrm{GeV}, f_{D_{s}}=0.258 \mathrm{GeV}, f_{K}=0.156 \mathrm{GeV}$, $f_{B}=0.188 \mathrm{GeV}$ and $f_{B_{c}}=0.436 \mathrm{GeV}$ [67]. $M_{N}$ and $\Gamma_{N}$ are the mass and decay width of the heavy neutrino $N$. Finally, the total decay rate is given by

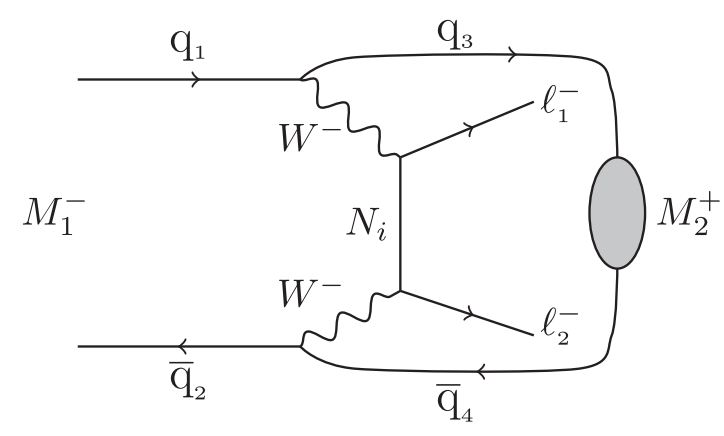

FIG. 2. The t-channel diagram for the lepton number violating meson decay. See the text for details.

$$
\Gamma\left(M_{1} \rightarrow \ell_{1} \ell_{2} \pi\right)=\frac{1}{n !} \sum|\mathcal{M}|^{2} d_{3}(\mathrm{PS})
$$

In Eq. (5), $n=2$ for identical final leptons, otherwise $n=1$ and $d_{3}(\mathrm{PS})$ is the three body phase space.

\section{TOTAL DECAY WIDTH OF $N$}

Although the RH neutrino $N$ is a SM singlet, due to mixing with active neutrinos it can decay via charged and neutral current interactions. For the mass range $0.140 \mathrm{GeV} \leq$ $M_{N} \leq 6 \mathrm{GeV}$, the RH neutrino can be produced as an intermediate on mass shell state in the LNV meson decays being considered here. We consider only tree level diagrams in the calculation of RH neutrino total decay width. In the relevant mass range $0.140 \mathrm{GeV} \leq M_{N} \leq 6 \mathrm{GeV}$, the following channels contribute to the total decay width of heavy neutrino:

(i) $N \rightarrow \ell^{-} P^{+}$, where $\ell=e, \mu, \quad \tau$, and $P^{+}=$ $\pi^{+}, K^{+}, D^{+}, D_{s}^{+}, B^{+}$(for $\ell=e, \mu$ ).

(ii) $N \rightarrow \nu_{\ell} P^{0}$, where $\nu_{\ell}$ are the flavor eigenstates $\nu_{e}$, $\nu_{\mu}, \nu_{\tau}$ and $P^{0}=\pi^{0}, \eta, \eta^{\prime}, \eta_{c}$.

(iii) $N \rightarrow \ell^{-} V^{+}$, where $\ell=e, \mu, \tau$, and $V^{+}=$ $\rho^{+}, K^{*+}, D^{*+}, D_{s}^{*+}, B^{*+}$ (for $\ell=e, \mu$ ).

(iv) $N \rightarrow \nu_{\ell} V^{0}$, where $\nu_{\ell}=\nu_{e}, \nu_{\mu}, \nu_{\tau}$ and $V^{0}=\rho^{0}, \omega, \phi$, $J / \psi$.

(v) $N \rightarrow \ell_{1}^{-} \ell_{2}^{+} \nu_{\ell_{2}}$, where $\ell_{1}, \ell_{2}=e, \mu, \tau, \ell_{1} \neq \ell_{2}$.

(vi) $N \rightarrow \nu_{\ell_{1}} \ell_{2}^{-} \ell_{2}^{+}$, where $\ell_{1}, \ell_{2}=e, \mu, \tau$.

(vii) $N \rightarrow \nu_{\ell_{1}} \nu \bar{\nu}$, where $\nu_{\ell_{1}}=\nu_{e}, \nu_{\mu}, \nu_{\tau}$.

Hence, the total decay width is given by

$$
\begin{aligned}
\Gamma_{N}= & \sum_{\ell, P^{+}} 2 \Gamma^{\ell P^{+}}+\sum_{\ell, P^{0}} \Gamma^{\nu_{\ell} P^{0}}+\sum_{\ell, V^{+}} 2 \Gamma^{\ell V^{+}}+\sum_{\ell, V^{0}} \Gamma^{\nu_{\ell} V^{0}} \\
& +\sum_{\ell_{1}, \ell_{2}\left(\ell_{1} \neq \ell_{2}\right)} 2 \Gamma^{\ell_{1} \ell_{2} \nu_{\ell_{2}}}+\sum_{\ell_{1}, \ell_{2}} \Gamma^{\nu_{\ell_{1}} \ell_{2} \ell_{2}}+\sum_{\nu_{\ell_{1}}} \Gamma^{\nu_{\ell_{1}} \nu \bar{\nu}} .
\end{aligned}
$$

As the RH neutrino is Majorana, the charge conjugate processes are also allowed and the decay rate is the same, hence the 2 factor is included for some of the channels. We can parametrize the above decay width as follows:

$$
\Gamma_{N}=a_{e}\left(M_{N}\right)\left|V_{e N}\right|^{2}+a_{\mu}\left(M_{N}\right)\left|V_{\mu N}\right|^{2}+a_{\tau}\left(M_{N}\right)\left|V_{\tau N}\right|^{2},
$$



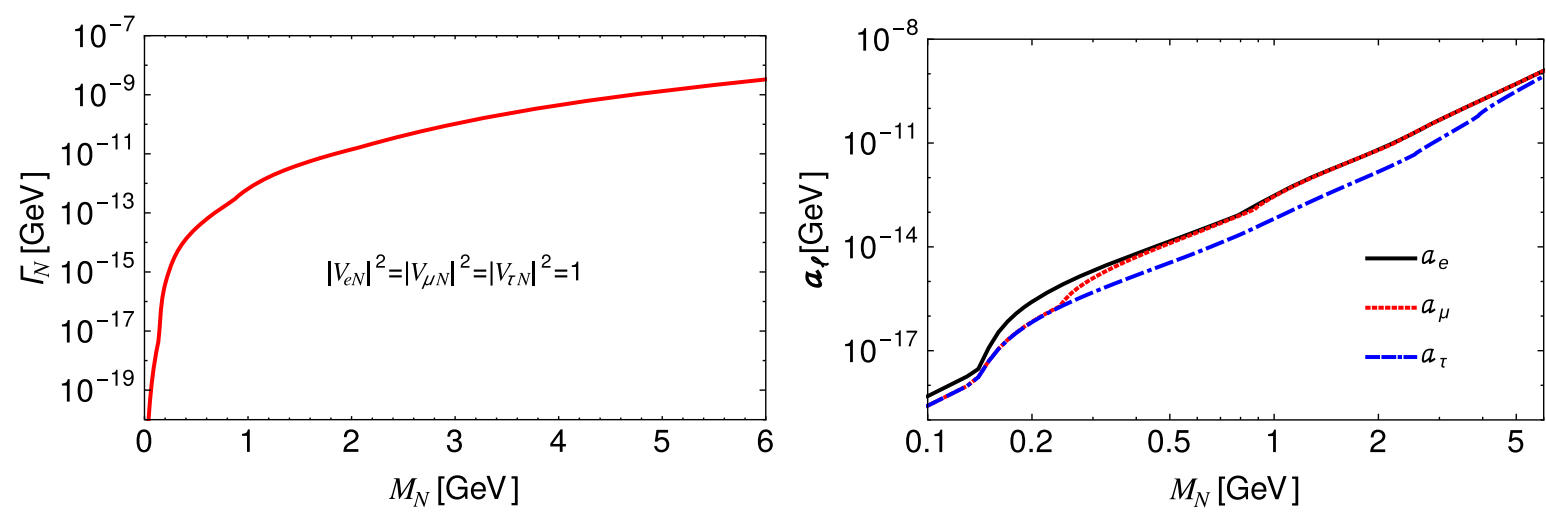

FIG. 3. Left panel: The total decay width of heavy neutrino $\mathrm{N}$ with the assumption, $\left|V_{e N}\right|^{2}=\left|V_{\mu N}\right|^{2}=\left|V_{\tau N}\right|^{2}=1$. Right panel: $a_{e}, a_{\mu}$ and $a_{\tau}$ as function of mass $M_{N}$.

where, $a_{e}, a_{\mu}$ and $a_{\tau}$ are functions of the Majorana neutrino mass and hence will differ from mode to mode. We show the total decay width of heavy neutrino $N$ in the left panel of Fig. 3, for the choice of mixing $\left|V_{e N}\right|^{2}=\left|V_{\mu N}\right|^{2}=$ $\left|V_{\tau N}\right|^{2}=1$. Even with mixing angle equal to 1 , the decay width lies in the range $10^{-16} \mathrm{GeV} \leq \Gamma_{N} \leq 10^{-7} \mathrm{GeV}$ for the $M_{N}$ mass range, $0.140 \mathrm{GeV} \leq M_{N} \leq 6 \mathrm{GeV}$. Hence, we can safely use the narrow-width approximation, $\frac{1}{\left(p_{N}^{2}-M_{N}^{2}\right)^{2}+M_{N}^{2} \Gamma_{N}^{2}} \approx$ $\frac{\pi}{M_{N} \Gamma_{N}} \delta\left(p_{N}^{2}-M_{N}^{2}\right)$ and $\Gamma\left(M_{1} \rightarrow \ell_{1} \ell_{2} M_{2}\right)$ can be approximated as $\Gamma\left(M_{1} \rightarrow \ell_{1} N\right) \operatorname{Br}\left(N \rightarrow \ell_{2} M_{2}\right)$. We also show in the right panel of Fig. 3 the different coefficients $a_{e}, a_{\mu}$ and $a_{\tau}$ as a function of RH neutrino mass $M_{N}$. For the RH neutrino mass $M_{N}$ up to $0.25 \mathrm{GeV}, a_{\mu} \approx a_{\tau}$ and for mass $M_{N}>0.4 \mathrm{GeV}, a_{e} \approx a_{\mu}$.

\section{PARENT MESON VELOCITY AND FINITE DETECTOR SIZE EFFECT}

For the mass range $0.140 \mathrm{GeV} \leq M_{N} \leq 6 \mathrm{GeV}$, the RH neutrinos produced in these LNV meson decays are on shell. The RH neutrino produced in meson decays $M_{1} \rightarrow \ell_{1} N$, propagates and decays after traveling some distance from the production point. This is the decay length $L_{N}$ of the RH neutrino $N$ and it depends on the total decay width of $N$. If $L_{N}$ is greater than the actual size of the detector, then $N$ will decay outside the detector and the signature $M_{1} \rightarrow \ell_{1} \ell_{2} \pi$ cannot be observed. For a particular experiment the detector size is finite. Hence, when calculating the signal events, we need to take into account this finite size detector effect by the probability factor $\mathcal{P}_{N}$ of $N$ to decay within the detector. In general, this probability factor can be written as

$\mathcal{P}_{N}=1-\exp \left(-L_{D} \Gamma_{N} \frac{M_{N}}{p_{N}}\right)=1-\exp \left(-\frac{L_{D}}{L_{N}}\right)$,

where $L_{N}=\frac{p_{N}}{M_{N} \Gamma_{N}}, L_{D}$ is the detector length, $p_{N}$ is the three momentum of $N$. Defining $x=\frac{L_{D}}{L_{N}}$, it is obvious that for a very large detector length $L_{D}$ and small decay length $L_{N}$,
$\mathcal{P}_{N}=1-\exp (-x) \approx 1$. Note that the probability factor depends on three momentum $p_{N}$, which in turn depends on the velocity of decaying meson $M_{1}$. Hence to incorporate the probability factor correctly, we need to use the correct velocity of the parent meson $M_{1}$ in each of the experiments. If the parent meson $M_{1}$ decays at rest, three momentum $p_{N}$ is fixed and is given by $p_{N}^{*}=\frac{m_{M_{1}}}{2} \lambda^{\frac{1}{2}}\left(1, \frac{m_{\ell}^{2}}{m_{M_{1}}^{2}}, \frac{M_{N}^{2}}{m_{M_{1}}^{2}}\right)$. For the case of parent meson $M_{1}$ produced with fixed boost $\vec{\beta}$, the energy of $N$ is then given by

$$
E_{N}=E_{N}^{*}\left(\gamma+\frac{p_{N}^{*}}{E_{N}^{*}} \sqrt{\gamma^{2}-1} \cos \theta_{N}^{*}\right)
$$

where $E_{N}^{*}$ is the energy of $N$ in the rest frame of $M_{1}$ which is given as $E_{N}^{*}=\sqrt{p_{N}^{* 2}+M_{N}^{2}} \cdot \gamma=\frac{E_{M_{1}}}{m_{M_{1}}}$ and $\theta_{N}^{*}$ is the emission angle of particle $N$ in the rest frame of $M_{1}$, which is measured from the boost direction $\vec{\beta}$. The energy $E_{N}$ of the $N$ in the boosted $M_{1}$ frame lies within the range

$$
\begin{gathered}
E_{N} \in\left[E_{N}^{-}, E_{N}^{+}\right]=\left[\left(\gamma E_{N}^{*}-p_{N}^{*} \sqrt{\gamma^{2}-1}\right),\right. \\
\left.\left(\gamma E_{N}^{*}+p_{N}^{*} \sqrt{\gamma^{2}-1}\right)\right] .
\end{gathered}
$$

Similarly we can derive the range of $p_{N} \in\left[p_{N}^{-}, p_{N}^{+}\right]$from Eq. (9) using the relation $p_{N}^{ \pm}=\sqrt{E_{N}^{ \pm 2}-M_{N}^{2}}$. In this section, we show how $x, L_{N}$ depends on parent meson velocity and compare to the case of parent meson decay at rest. For the case of meson decay at rest, $p_{N}=p_{N}^{*}$ and for meson decay with nonzero momentum $p_{M_{1}}$, we take $p_{N}=\frac{p_{N}^{-}+p_{N}^{+}}{2}$ to compare. With the assumption of $\left|V_{e N}\right|^{2}=\left|V_{\mu N}\right|^{2}=$ $\left|V_{\tau N}\right|^{2}$, we can write the decay length $L_{N}$ and $x$ as

$L_{N}=\frac{p_{N}}{M_{N} \Gamma_{N}}=\frac{p_{N}}{M_{N}\left|V_{\ell N}\right|^{2}\left(a_{e}\left(M_{N}\right)+a_{\mu}\left(M_{N}\right)+a_{\tau}\left(M_{N}\right)\right)}$ 

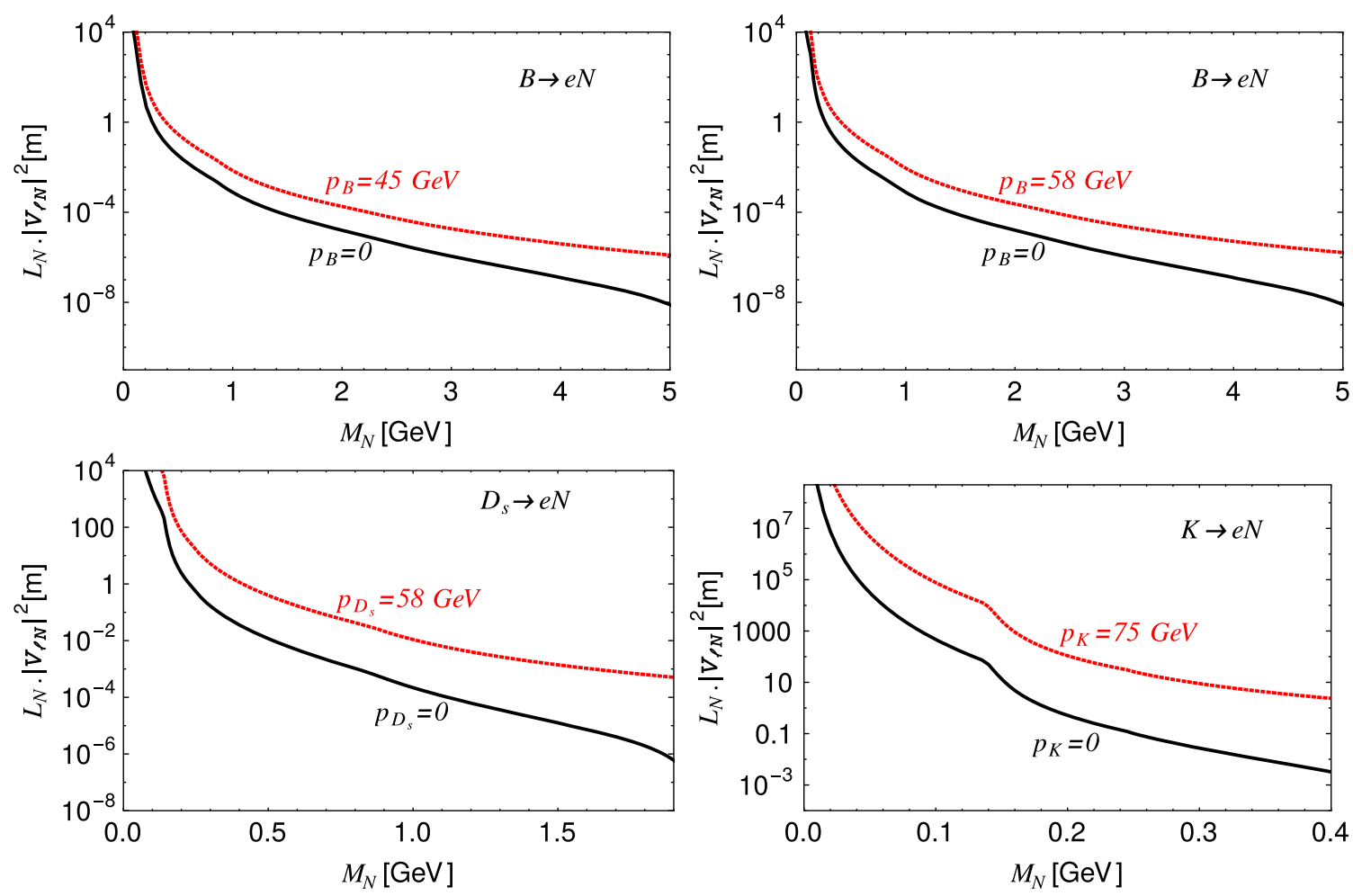

FIG. 4. Dependence of the decay length $L_{N}$ on parent meson velocity as a function of RH neutrino mass $M_{N}$. The upper panel is for $B$ meson decay at Belle-II (left) and SHiP (right). The left figure of the lower panel is for $D_{s}$ meson decay at SHiP and the right figure of the lower panel is for $K$ meson decay at NA62.
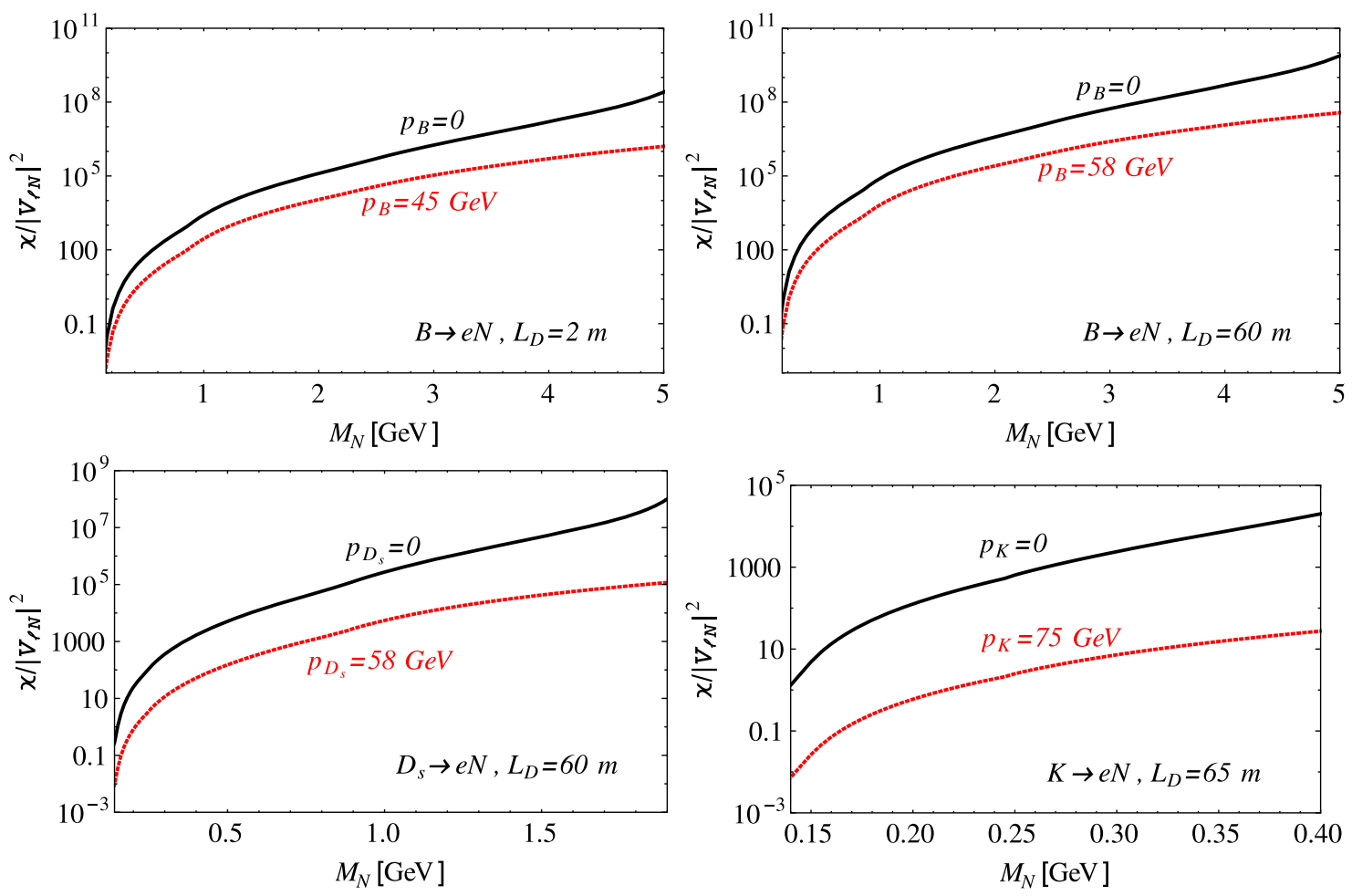

FIG. 5. Dependence of parameter $x=\frac{L_{D}}{L_{N}}$ on parent meson velocity as a function of RH neutrino mass $M_{N}$. The upper panel is for $B$ meson decay at Belle-II (left) and SHiP (right). The left figure of the lower panel is for $D_{s}$ meson decay at SHiP and the right figure of the lower panel is for $K$ meson decay at NA62. 
$x=\frac{L_{D}}{L_{N}}=\frac{L_{D}}{p_{N}} M_{N}\left|V_{\ell N}\right|^{2}\left(a_{e}\left(M_{N}\right)+a_{\mu}\left(M_{N}\right)+a_{\tau}\left(M_{N}\right)\right)$.

In Figs. 4 and 5, we have shown the variations of $L_{N} \times$ $\left|V_{\ell N}\right|^{2}$ and $\frac{x}{\left|V_{\ell N}\right|^{2}}$ as a function of RH neutrino mass $M_{N}$ in $B$, $D_{s}$ and $K$ meson decays. To do the comparison with the meson decay at rest $\left(p_{M_{1}}=0\right)$, we take $p_{B}=45 \mathrm{GeV}$ (FCC-ee) [68], $58 \mathrm{GeV}$ (SHiP) [69]; $p_{D_{s}}=58 \mathrm{GeV}$ (SHiP) and $p_{K}=75 \mathrm{GeV}$ (NA62) [70]. From these two figures it is clear that decay length increases (hence $x$ decreases) for fixed mixing angle in the case of meson decays in flight compared to meson decay at rest. Hence, the probability of RH neutrino $\mathcal{P}_{N}$ to decay inside the detector is smaller in the case of meson decay in flight compared to meson decay at rest. As a result, compare to meson decay at rest, in the case of meson decay in flight we get a rather loose bound on mixing angle from the expected signal events.

\section{SIGNAL EVENTS}

The sensitivity reach for the LNV decay modes in a particular experiment depends on the number of the parent mesons $M_{1}$ 's produced $\left(N_{M_{1}^{-}}\right)$, their momentum $\left(\vec{p}_{M_{1}}\right)$ and the branching ratio for these mesons to the LNV modes. Assuming the parent meson $M_{1}$ decays at rest, the expected number of signal events is [71]:

$$
\begin{aligned}
N_{\text {event }} & =2 N_{M_{1}^{-}} \operatorname{Br}\left(M_{1}^{-} \rightarrow \ell_{1}^{-} \ell_{2}^{-} M_{2}^{+}\right) \mathcal{P}_{N}, \\
& \approx 2 N_{M_{1}^{-}} \operatorname{Br}\left(M_{1}^{-} \rightarrow \ell_{1}^{-} N\right) \frac{\Gamma\left(N \rightarrow \ell_{2}^{-} M_{2}^{+}\right)}{\Gamma_{N}} \mathcal{P}_{N},
\end{aligned}
$$

the factor 2 is due to inclusion of the charge conjugate process $M_{1}^{+} \rightarrow \ell_{1}^{+} N$ and $\mathcal{P}_{N}$ is the detector probability which is given by

$$
\mathcal{P}_{N}=\left[1-\exp \left(-\frac{M_{N} \Gamma_{N} L_{D}}{p_{N}^{*}}\right)\right]
$$

For the case of meson decay in flight the RH neutrino energy $E_{N}$ lies in range according to Eq. (9) and follows a flat distribution as

$$
f\left(E_{N}\right)=\frac{1}{E_{N}^{+}-E_{N}^{-}}=\frac{1}{2 p_{N}^{*} \sqrt{\gamma^{2}-1}} .
$$

Hence to calculate the total number of events for $M_{1}^{-} \rightarrow$ $\ell_{1}^{-} \ell_{2}^{-} M_{2}^{+}$in the lab frame we need to integrate within the range of $E_{N}$ as

$$
\begin{aligned}
N_{\text {event }} \approx & 2 N_{M_{1}^{-}} \int_{E_{N}^{-}}^{E_{N}^{+}} d E_{N} \operatorname{Br}\left(M_{1}^{-} \rightarrow \ell_{1}^{-} N\right) \frac{m_{M_{1}}}{2 p_{N}^{*}\left|\vec{p}_{M_{1}}\right|} \\
& \times \frac{\Gamma\left(N \rightarrow \ell_{2}^{-} M_{2}^{+}\right)}{\Gamma_{N}} \mathcal{P}_{N}^{\prime},
\end{aligned}
$$

where $\mathcal{P}_{N}^{\prime}=\left[1-\exp \left(-\frac{M_{N} \Gamma_{N} L_{D}}{\sqrt{E_{N}^{2}-M_{N}^{2}}}\right)\right]$ is the detector probability after taking into account the parent meson $M_{1}$ velocity.

Since the LNV meson decay rates will be extremely small, the expected number of signal events for these processes can be assumed to follow a Poisson distribution. Following Ref. [72] and assuming zero background events, we derive the average upper limit on the number of events at $95 \%$ C.L., assuming the number of signal events to be $N_{\text {event }}=3.09$.

Note that the number of events given in Eq. (12) or (13) are functions of the mass parameters $M_{N}$ and mixing $V_{\ell N}$. Equating the numerical upper limit on the number of events to the theoretical expressions, we get constraints on mixing angle $V_{\ell N}$, corresponding to specific $M_{N}$ values for particular experiments. We have assumed $\left|V_{e N}\right|^{2}=$ $\left|V_{\mu N}\right|^{2}=\left|V_{\tau N}\right|^{2}$ in $\Gamma_{N}$ when deriving these bounds using Eqs. (12) and (13).

\section{INPUT FOR DIFFERENT EXPERIMENTS}

\section{A. $\mathbf{L H C b}$}

The $\mathrm{LHCb}$ detector is a forward spectrometer at the Large Hadron Collider (LHC) at CERN. A search for heavy Majorana neutrinos in $B \rightarrow \mu \mu \pi$ decay mode had been performed by the $\mathrm{LHCb}$ collaboration using $7 \mathrm{TeV}$ data [73] and bound on the mixing angle $\left|V_{\mu N}\right|^{2}$ is provided in the mass range $0.25 \mathrm{GeV} \leq M_{N} \leq 5 \mathrm{GeV}$. ${ }^{1}$ The cross section for producing $\mathrm{B}, D$ and $D_{s}$ mesons at $\sqrt{s}=$ $13 \mathrm{TeV}$ within the LHCb acceptance $(2<\eta<5)$ is 86.6, 834 and $353 \mu \mathrm{b}$, respectively [75,76]. Hence, in $\mathrm{LHCb}$ upgrade with $300 \mathrm{fb}^{-1}$ luminosity, the expected number of $\mathrm{B}, D$ and $D_{s}$ mesons is $N_{B^{+}}=2.6 \times 10^{13}, N_{D^{+}}=2.5 \times$ $10^{14}$ and $N_{D_{s}^{+}}=1.05 \times 10^{14}$. LHCb will also produce a large number of $B_{c}$ mesons. A crude estimate [77] using the measured [78] ratio of production cross section times branching fractions between the $B_{c} \rightarrow J / \psi \pi^{+}$and $B^{+} \rightarrow$ $J / \psi K^{+}$decays at $\sqrt{s}=8 \mathrm{TeV}$ indicates $\mathcal{O}\left(10^{11}\right) B_{c}$ events with $300 \mathrm{fb}^{-1}$ luminosity at $14 \mathrm{TeV}$. Though the number of $B_{c}$ mesons at $\mathrm{LHCb}$ are smaller than the number of $B$ mesons, this mode being less suppressed with respect to $B^{+} \rightarrow \ell_{1}^{+} \ell_{2}^{+} \pi^{-}$gives tighter constraints on the mixing angles. The produced mesons will decay in flight, carrying a momentum of the order of $100 \mathrm{GeV}$ in the forward direction [79]. We take the detector length $L_{D} \approx 20 \mathrm{~m}$.

\footnotetext{
${ }^{1}$ This bound has been recently revised in Ref. [74] by taking into account the correct lifetime calculation of $N$.
} 


\section{B. NA62}

NA62 is an ongoing experiment at CERN that will produce a large number of $K^{+}$mesons [70]. The primary SPS $400 \mathrm{GeV}$ proton beam aims on a target and produces a secondary high intensity hadron beam with an optimum content of $K^{+}(\approx 6 \%)$. The expected number of $K^{+}$decays in the fiducial volume is $4.5 \times 10^{12}$ per year. Assuming three years of running, $N_{K^{+}}=1.35 \times 10^{13}$. The detector length $L_{D} \approx 65 \mathrm{~m}$ and the produced $K^{+}$mesons will decay in flight, carrying a momentum of $75 \mathrm{GeV}$.

\section{SHiP}

The SHiP experiment is a newly proposed general purpose fixed target facility at the CERN SPS accelerator [80]. A $400 \mathrm{GeV}$ proton beam will be dumped on a heavy target for the duration of five years. One of the primary goals of the experiment is to use decays of charmed mesons to search for heavy sterile neutrinos using the decay mode $D_{s}^{+} / D^{+} \rightarrow \ell^{+} \ell^{+} \pi^{-}$. One can easily estimate the number of charmed meson pairs that are expected to be produced in this experiment as [81]

$$
N_{\text {meson }}=X_{c \bar{c}} \times N_{\text {POT }} \times \mathcal{R},
$$

where $X_{c \bar{c}}$ is the $c \bar{c}$ production rate, $N_{\mathrm{POT}}=2 \times 10^{20}$ is the number of proton-target interaction. The relative abundances $\mathcal{R}$ of charmed mesons, such as $D$ and $D_{s}$, are $30 \%$ and $8 \%$, respectively. Hence, the expected number of $D$ and $D_{s}$ mesons are $N_{D^{+}}=1.02 \times 10^{17}$ and $N_{D_{s}^{+}}=2.72 \times 10^{16}$, respectively. This very high intensity of the charmed mesons will permit to set tight constraints on mixing angle at SHiP. There will also be a large number of $B$ and $B_{c}$ meson productions at SHiP. Following [82], we can estimate the number of $B$ and $B_{c}$ meson as $N_{B^{+}}=10^{13}$ and $N_{B_{c}}=10^{11}$, respectively. The detector length is taken to be $L_{D}=60 \mathrm{~m}$. For the $400 \mathrm{GeV}$ CERN Neutrinos to Gran Sasso proton beam on target, the expected momentum of the produced mesons is $\sim 58 \mathrm{GeV}$ [69].

\section{MATHUSLA}

MATHUSLA [83] is a newly proposed detector near ATLAS or CMS. Its main goal is to search for neutral longlived particles produced in HL-LHC collisions by reconstructing displaced vertices. The detector is designed to have an area of $200 \mathrm{~m} \times 200 \mathrm{~m}$ and a height of $20 \mathrm{~m}$ for the decay volume, which is displaced from ATLAS or CMS by $100 \mathrm{~m}$ both horizontally and vertically. RH neutrino search is one of the primary goal of MATHUSLA and is most sensitive to the parameter space which yields a decay length $\sim 200 \mathrm{~m}$. RH neutrinos which are coming from the meson decays or $W$ and $Z$ boson decays has very large decay length and this has been already studied in [83]. For the meson decay case, they have considered the decay modes $B \rightarrow D \ell N, B \rightarrow \ell N$ and $D \rightarrow K \ell N$ and after including the probability of RH neutrinos to decay visibly within the MATHUSLA detector, they derived the constraints on mixing angles. In this study, we have considered the meson decays $B \rightarrow \ell_{1} \ell_{2} \pi$ and $D \rightarrow \ell_{1} \ell_{2} \pi$ for MATHUSLA. For the number of $B$ and $D$ meson productions we followed Ref. [84]. The result of their detailed simulation suggests that numbers of $B$ and $D$ meson production within the geometric acceptance of the MATHUSLA detector are $5.7 \times 10^{14}$ and $5.4 \times 10^{13}$, respectively. Their simulation also gives the average $\gamma$ factor of the $B$ and $D$ mesons as $\left\langle\gamma_{B}\right\rangle=2.3$ and $\left\langle\gamma_{D}\right\rangle=2.6$ from which we can derive the average momentum of the mesons. The detector length is taken to be $38 \mathrm{~m}$.

\section{E. Belle II}

The asymmetric SuperKEKB facility is designed to collide electron and positron beams such that the center of mass energy is in the region of the $\Upsilon$ resonances. An upgrade of Belle, the newly completed Belle II detector is expected to collect data samples corresponding to an integrated luminosity of $50 \mathrm{ab}^{-1}$ by the end of 2024 [85]. The expected number of charged $B \bar{B}$ pairs to be produced is $5.5 \times 10^{10}[86,87]$. In addition, a large sample of charged $D, D_{s}$ mesons will also be accessible, with $N_{D^{+}}=3.4 \times$ $10^{10}$ and $N_{D_{s}^{+}}=10^{10}$ [87]. A direct search for heavy Majorana neutrinos in B-meson decays was performed by the Belle collaboration using a data sample that contained $772 \times 10^{6} B \bar{B}$ pairs (at $711 \mathrm{fb}^{-1}$ ) [88]. At KEKB as well as SuperKEKB, the energies of the $e^{+}, e^{-}$beams are sufficiently low so that the momentum of the produced $B$ mesons as well as that for the charmed mesons will not be appreciable and the suppression from high momentum of the decaying mesons in the number of events will be absent.

\section{F. FCC-ee}

The Future Circular Collider (FCC-ee) [68] will collect multi-ab ${ }^{-1}$ integrated luminosities for $e^{+} e^{-}$collisions at c.m. energy $\sqrt{s} \approx 91 \mathrm{GeV}$. The expected number of $Z$-bosons is $10^{12}-10^{13}$. The number of charged $\mathrm{B}$ mesons from $Z$ decays can be estimated as

$$
N_{B^{+}}=N_{Z} \times \operatorname{Br}(Z \rightarrow b \bar{b}) \times f_{u},
$$

where $N_{Z} \sim 10^{13}, \operatorname{Br}(Z \rightarrow b \bar{b})=0.1512$ [89], $f_{u}=0.410$ [90] is the fraction of $B^{+}$from $\bar{b}$ quark in $Z$ decay. The $\mathrm{B}$ mesons produced at FCC-ee will have an energy distribution peaked at $E_{B^{+}}=\frac{M_{Z}}{2}$. Hence we can calculate the number of signal events using Eq. (13), where the detector length is taken to be $L_{D}=2 \mathrm{~m}$.

\section{RESULTS}

In Fig. 6, we show how the velocity of the parent mesons affects the sensitivity reach of the mixing angles. 

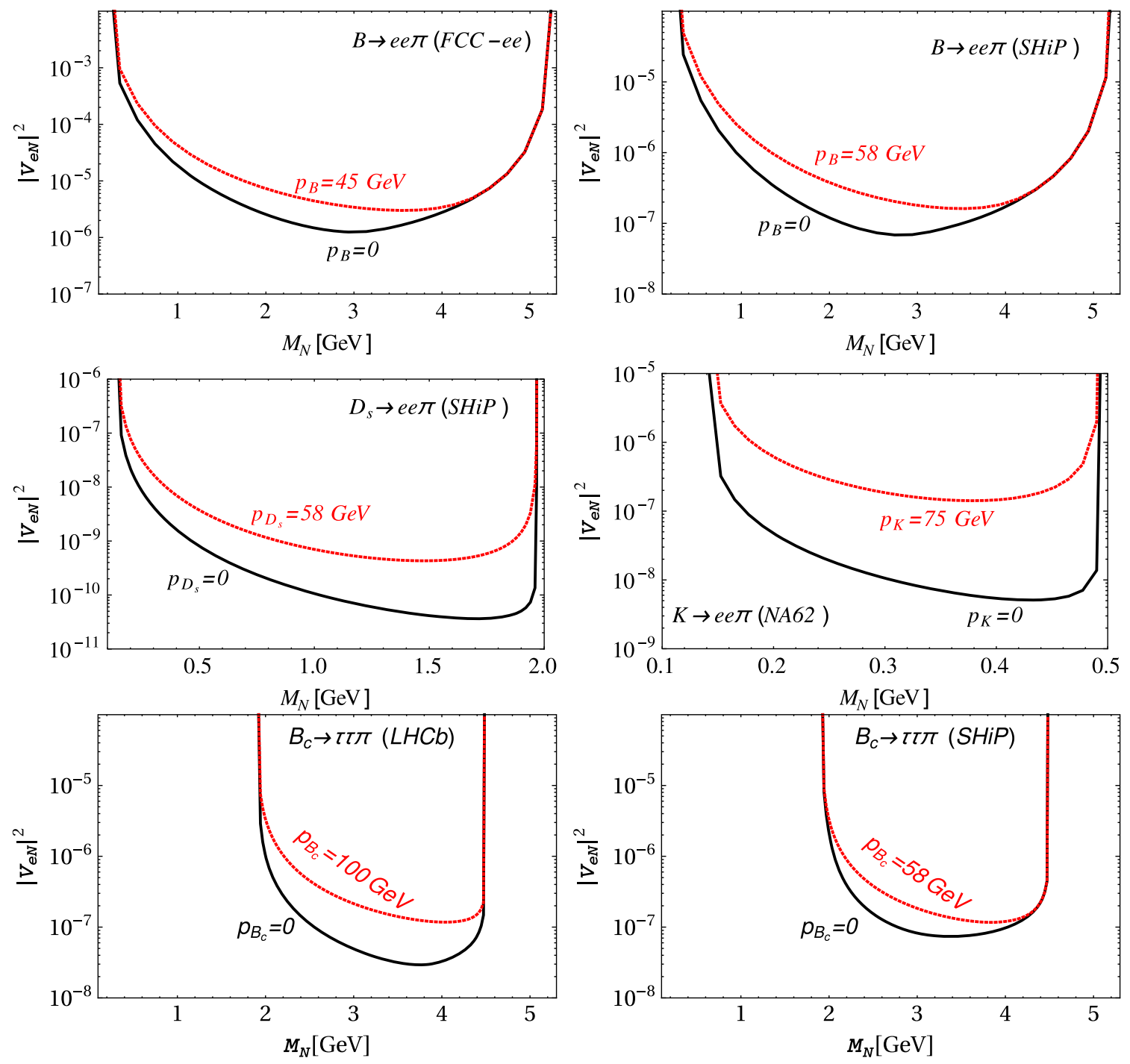

FIG. 6. Future sensitivity reach and present limits on the mixing angles as a function of RH neutrino mass $M_{N}$. The black and red lines stand for meson decay at rest and meson decay with finite momentum, respectively. The upper panel corresponds to the $B$ meson decay at FCC-ee (left) and SHiP (right). The left figure of the middle panel is for $D_{s}$ meson decay at SHiP and the right figure of the middle panel is for $K$ meson decay at NA62. The lower panel represents $B_{c}$ meson decay at LHCb (left) and SHiP (right).

We consider a number of ongoing and future experiments, such as FCC-ee, SHiP to explore $B \rightarrow e e \pi$, SHiP for $D_{s} \rightarrow e e \pi$, NA62 for $K \rightarrow e e \pi$, and LHCb, SHiP for $B_{c} \rightarrow \tau \tau \pi$ meson decays. To derive the bounds/sensitivity on the mixing angle as a function of $\mathrm{RH}$ neutrino mass $M_{N}$, we use Eqs. (12) and (13), for meson decay at rest and in flight, respectively. For all of the above decays, the obtained bounds/future sensitivity on the mixing angles are rather loose in the case of meson decays in flight compared to meson decays at rest. As an example, for the case of $B$ (FCC-ee, SHiP) and $D_{s}$ (SHiP) meson decays, there is approximately 1 order of magnitude difference between the two results. The result for $K$ meson decay at NA62 differs by 2 orders of magnitude. Hence, the inclusion of parent meson velocity is indeed very important when calculating the bounds on the mixing angles.

In Figs. 7-10, we show the final bounds and future sensitivity on various mixing angles such as $\left|V_{\ell N}\right|^{2}, \ell=e$, $\mu, \tau$ and $\left|V_{\ell_{1} N} V_{\ell_{2} N}\right|, \ell_{1}, \ell_{2}=e, \mu, \tau, \ell_{1} \neq \ell_{2}$ as a function of RH neutrino mass $M_{N}$. When calculating the limits on these mixing angles, we are using Eq. (12) for Belle-II and Eq. (13) for the other experiments. The latter properly takes into account parent meson velocity effect in the $\mathrm{RH}$ neutrino decay probability inside the detector. Due to the huge number of charmed meson productions, the future experiment SHiP will be able to probe $\left|V_{e N}\right|^{2},\left|V_{\mu N}\right|^{2} \sim$ $\mathcal{O}\left(10^{-9}\right)$ and $\left|V_{e N} V_{\mu N}\right| \sim \mathcal{O}\left(10^{-9}\right)$ in the mass range $0.14 \mathrm{GeV}<M_{N}<1.9 \mathrm{GeV}$. Figure 6 shows that without 

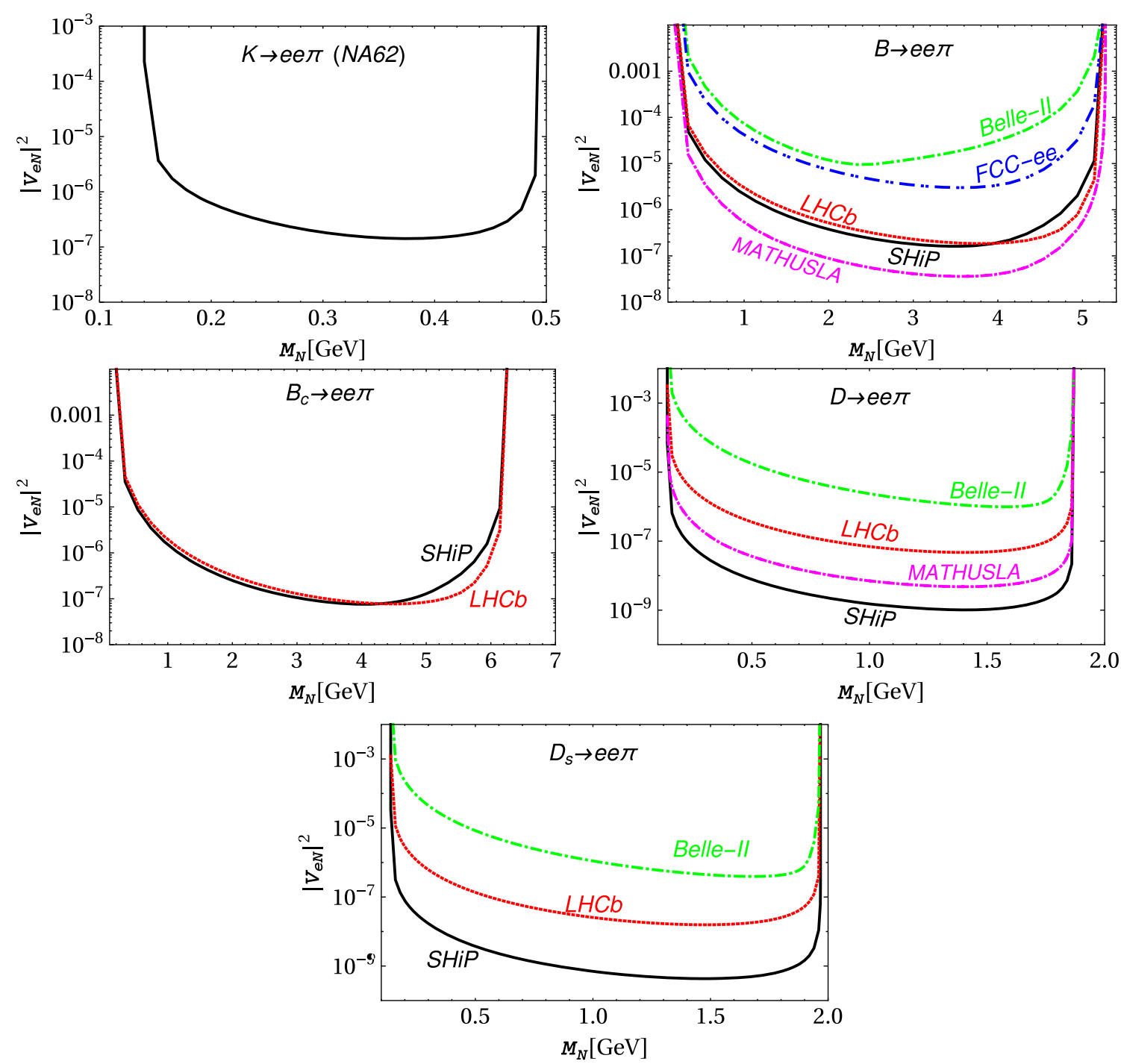

FIG. 7. Variation of the future sensitivity reach and present limits on the mixing angle $\left|V_{e N}\right|^{2}$ with respect to the mass $M_{N}$. We consider meson decay channel $M_{1} \rightarrow e e \pi$. The left figure in the upper panel is for $K$ meson decay at NA62. The right figure in the upper panel is for $B$ meson decay at SHiP (black), MATHUSLA (magenta), LHCb (red), FCC-ee (blue) and Belle-II (green). The left figure in the middle panel is for $B_{c}$ meson decay at SHiP (black) and $\mathrm{LHCb}$ (red). The right figure of the middle panel is for $D$ meson decay at SHiP (black), MATHUSLA (magenta), LHCb (red) and Belle-II (green). The last figure is for $D_{s}$ meson decay at SHiP (black), LHCb (red) and Belle-II (green).

considering the $K$ meson velocity, tightest bounds on mixing angles $\left|V_{e N}\right|^{2} \quad\left(0.14 \mathrm{GeV}<M_{N}<0.49 \mathrm{GeV}\right)$, $\left|V_{\mu N}\right|^{2} \quad\left(0.24 \mathrm{GeV}<M_{N}<0.38 \mathrm{GeV}\right)$ and $\left|V_{e N} V_{\mu N}\right|$ $\left(0.14 \mathrm{GeV}<M_{N}<0.49 \mathrm{GeV}\right)$ are obtained from the meson decays $K \rightarrow e e \pi, \quad K \rightarrow \mu \mu \pi$ and $K \rightarrow e \mu \pi$, respectively. Taking into account parent meson velocity, the tightest bound in the above mass ranges can instead be obtained from the $D_{s}$ meson decays at SHiP. For relatively higher mass range $2 \mathrm{GeV}<M_{N}<5 \mathrm{GeV}$, the tightest bound on mixing angles $\left|V_{e N}\right|^{2}, \quad\left|V_{\mu N}\right|^{2} \sim$ $\mathcal{O}\left(10^{-7}\right)$ and $\left|V_{e N} V_{\mu N}\right| \sim \mathcal{O}\left(10^{-7}\right)$ can be obtained from $B$ meson decays at MATHUSLA. Finally for the mass range $5 \mathrm{GeV}<M_{N}<6 \mathrm{GeV}$, the tightest limit on mixing angles $\left|V_{e N}\right|^{2},\left|V_{\mu N}\right|^{2} \sim \mathcal{O}\left(10^{-7}\right)$ and $\left|V_{e N} V_{\mu N}\right| \sim$ $\mathcal{O}\left(10^{-7}\right)$ will be provided by the $B_{c}$ meson decay at $\mathrm{LHCb}$.

Furthermore, the large mass gap between $B\left(B_{c}\right)$ and $\pi$ meson allows one or both final leptons to be tau. Hence, we have included in our study additional final states like $B, B_{c} \rightarrow e \tau \pi, \mu \tau \pi$ and $\tau \tau \pi$. The highest sensitivity reach on $\left|V_{e N} V_{\tau N}\right| \sim \mathcal{O}\left(10^{-7}\right)$ and $\left|V_{\mu N} V_{\tau N}\right| \sim \mathcal{O}\left(10^{-7}\right)$ can be provided from the $B \rightarrow e(\mu) \tau \pi \quad\left(0.2 \mathrm{GeV}<M_{N}<\right.$ $5 \mathrm{GeV})$ at MATHUSLA and $B_{c} \rightarrow e(\mu) \tau \pi\left(5 \mathrm{GeV}<M_{N}<\right.$ $6 \mathrm{GeV})$ at $\mathrm{LHCb}$, respectively. Additionally, the tightest bound on $\left|V_{\tau N}\right|^{2} \sim \mathcal{O}\left(2 \times 10^{-7}\right)$ can also be provided by $B \rightarrow \tau \tau \pi$ decay mode $\left(2 \mathrm{GeV}<M_{N}<3.4 \mathrm{GeV}\right)$ 

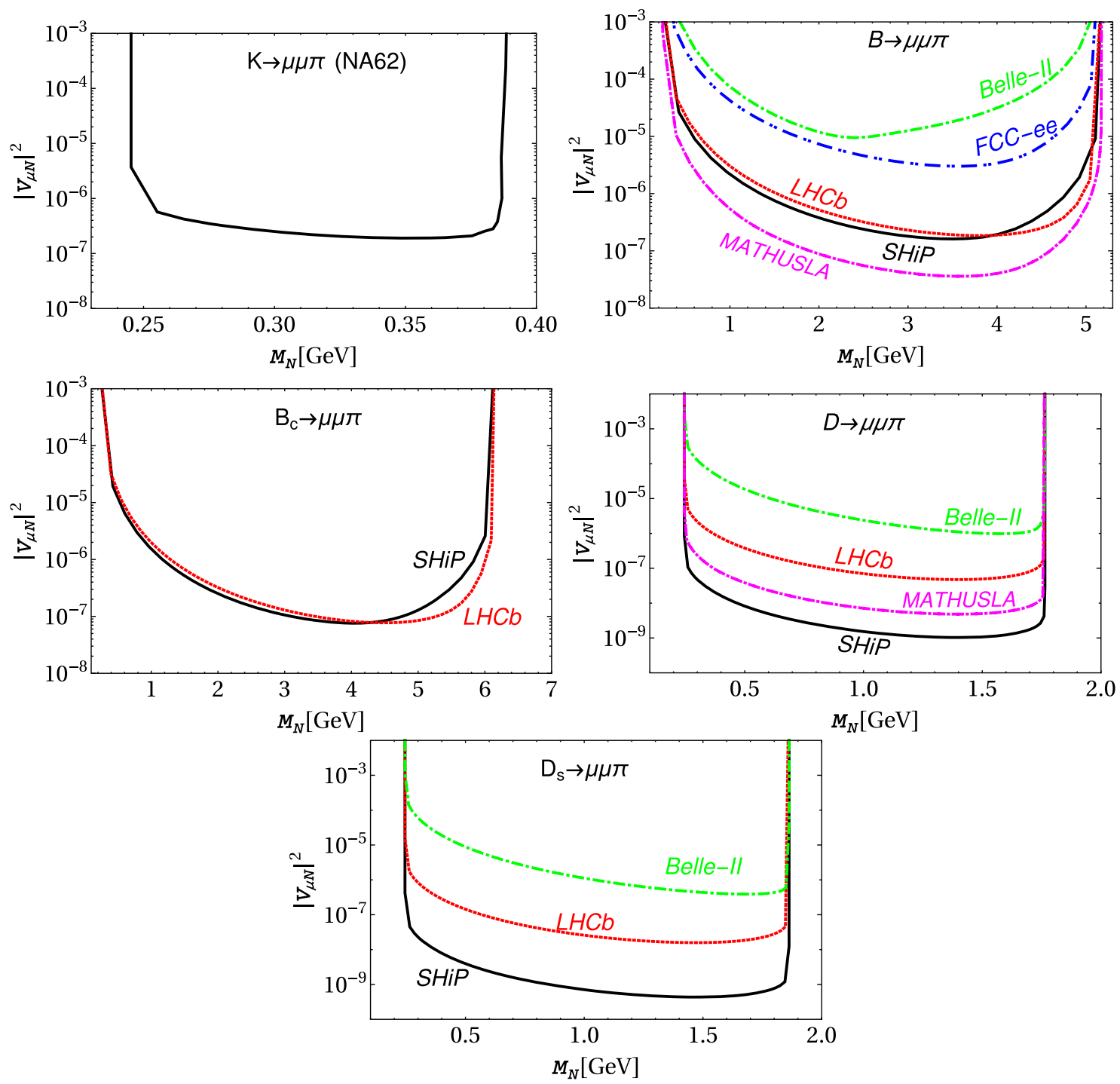

FIG. 8. Same as Fig. 7 except now the limits are on the mixing angle $\left|V_{\mu N}\right|^{2}$ from the meson decays $M_{1} \rightarrow \mu \mu \pi$.

at MATHUSLA and $B_{c} \rightarrow \tau \tau \pi$ decay mode $\left(3.4 \mathrm{GeV}<M_{N}<4.5 \mathrm{GeV}\right)$ at $\mathrm{LHCb}$, respectively. Note that $B, B_{c} \rightarrow \tau \tau \pi$ meson decays constrain the mixing angle $\left|V_{\tau N}\right|^{2}$ in the mass range, where it has so far been unconstrained by any of the $\tau$ or other meson decays. In spite of the larger number of $D$ production, compared to $D_{s}$ meson at SHiP, the suppression from the weak annihilation vertex in the case of $D_{s}$ meson $V_{c s}$ is less compared to $D$ meson $V_{c d}$. As a result of this, tightest limits on the mixing angles will be provided by the $D_{s}$ meson decays in the relatively lower mass range.

Note that, if both of the like sign dileptons are not of the same flavor $\left(\ell_{1} \neq \ell_{2}\right)$, then the process is not only lepton number violating, but also lepton flavor violating. Further, if the distance between $N$ production and decay points is large enough, then the two processes, $M_{1} \rightarrow$ $\ell_{1} N$ followed by $N \rightarrow \ell_{2} \pi$, will be separated. Assuming this separation, the two processes $M_{1} \rightarrow \ell_{1} \ell_{2} \pi$ and $M_{1} \rightarrow \ell_{2} \ell_{1} \pi$ can be distinguished. While deriving the bounds on the mixing angles for the case of $\ell_{1} \neq \ell_{2}$, we are assuming this separation in our study. This is justified as the decay width $\Gamma_{N}$ is very small (hence the lifetime is very large) in the mass range of interest. The allowed mass range of $N$ for the decay modes $M_{1} \rightarrow \ell_{1} \ell_{2} \pi$ and $M_{1} \rightarrow \ell_{2} \ell_{1} \pi$ are $m_{\ell_{2}}+m_{\pi}<M_{N}<m_{M_{1}}-m_{\ell_{1}}$ and $m_{\ell_{1}}+m_{\pi}<M_{N}<m_{M_{1}}-m_{\ell_{2}}$, respectively. We consider both of the channels $M_{1} \rightarrow \ell_{1} \ell_{2} \pi$ and $M_{1} \rightarrow \ell_{2} \ell_{1} \pi$ to derive the bound on the mixing angle $\left|V_{\ell_{1} N} V_{\ell_{2} N}\right|$.

One important point to note is that we have considered an idealized detector with $100 \%$ detection, reconstruction efficiencies etc. to derive the constraints on the mixing angles. The realistic constraints are expected to be weaker and will only be feasible through searches by the experimental collaborations, incorporating the detection, reconstruction efficiencies in the actual experiment. 

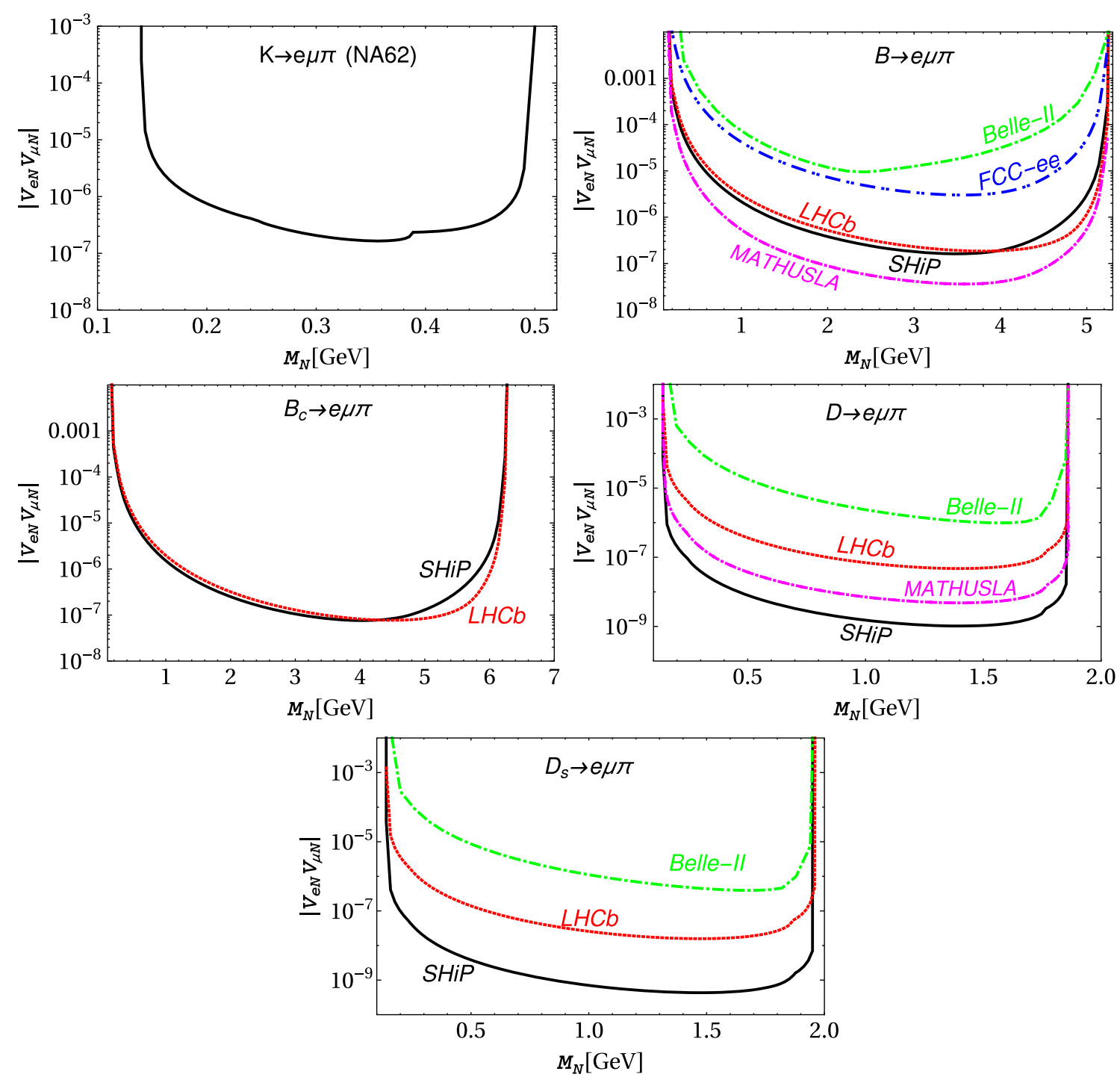

FIG. 9. Same as Fig. 7. The plots in different panels show the sensitivity reach of the mixing angle $\left|V_{e N} V_{\mu N}\right|$ from the meson decays $M_{1} \rightarrow e \mu \pi$.

\section{COMBINED SENSITIVITY REACH FROM MESON DECAYS AND COMPARISON WITH EXISTING CONSTRAINTS}

In this section, we discuss the future sensitivity reach from LNV three body meson decays. The combined limits represent the strongest limits obtained in different mass ranges of $N$. In Fig. 11, we show the combined sensitivity reach for $\left|V_{e N}\right|^{2}$ by the dark blue solid line. This corresponds to the tightest constraints that can be obtained from $D_{s} \rightarrow e e \pi$ mode (by SHiP) in the lower mass range $0.14 \mathrm{GeV}<M_{N}<2 \mathrm{GeV}$, and from $B \rightarrow e e \pi$ (by MATHUSLA), $B_{c} \rightarrow e e \pi$ (by LHCb) in the higher mass range $2 \mathrm{GeV}<M_{N}<6 \mathrm{GeV}$. Note that, for the lower mass range, SHiP can probe $\left|V_{e N}\right|^{2} \sim 10^{-9}$, while for higher mass range, MATHUSLA and $\mathrm{LHCb}$ can probe $\left|V_{e N}\right|^{2} \sim 10^{-7}$. In particular, the very near future accumulation of data $\left(\mathcal{L}=300 \mathrm{fb}^{-1}\right)$ in LHCb can probe $\left|V_{e N}\right|^{2} \sim 10^{-7}$, around $M_{N} \sim 5 \mathrm{GeV}$. The sensitivity reach of $\left|V_{\mu N}\right|^{2}$, as shown in Fig. 12, is very similar. The combined limit represents the constraint from $D_{s} \rightarrow \mu \mu \pi, B \rightarrow \mu \mu \pi$ and $B_{c} \rightarrow \mu \mu \pi$ decay modes that can again be probed in SHiP, MATHUSLA and LHCb. For $\left|V_{\tau N}\right|^{2}$, the best sensitivity reach $\left|V_{\tau N}\right|^{2} \sim 10^{-7}$ can be provided by MATHUSLA in $B \rightarrow \tau \tau \pi$ mode, while SHiP and $\mathrm{LHCb}$ can give similar sensitivity reach with the mode $B_{c} \rightarrow \tau \tau \pi$. The combined sensitivity reach has been shown in Fig. 13.

The future sensitivity of $\left|V_{e N} V_{\mu N}\right|,\left|V_{\mu N} V_{\tau N}\right|$ and $\left|V_{e N} V_{\tau N}\right|$ are shown in Figs. 14, 15 and 16, respectively. For $\left|V_{e N} V_{\mu N}\right|$ mode, the lower mass range up to $M_{N} \sim$ $2 \mathrm{GeV}$ can be probed by the channel $D_{s} \rightarrow e \mu \pi$ (by SHiP) where sensitivity down to $\left|V_{e N} V_{\mu N}\right| \sim 10^{-9}$ can be 

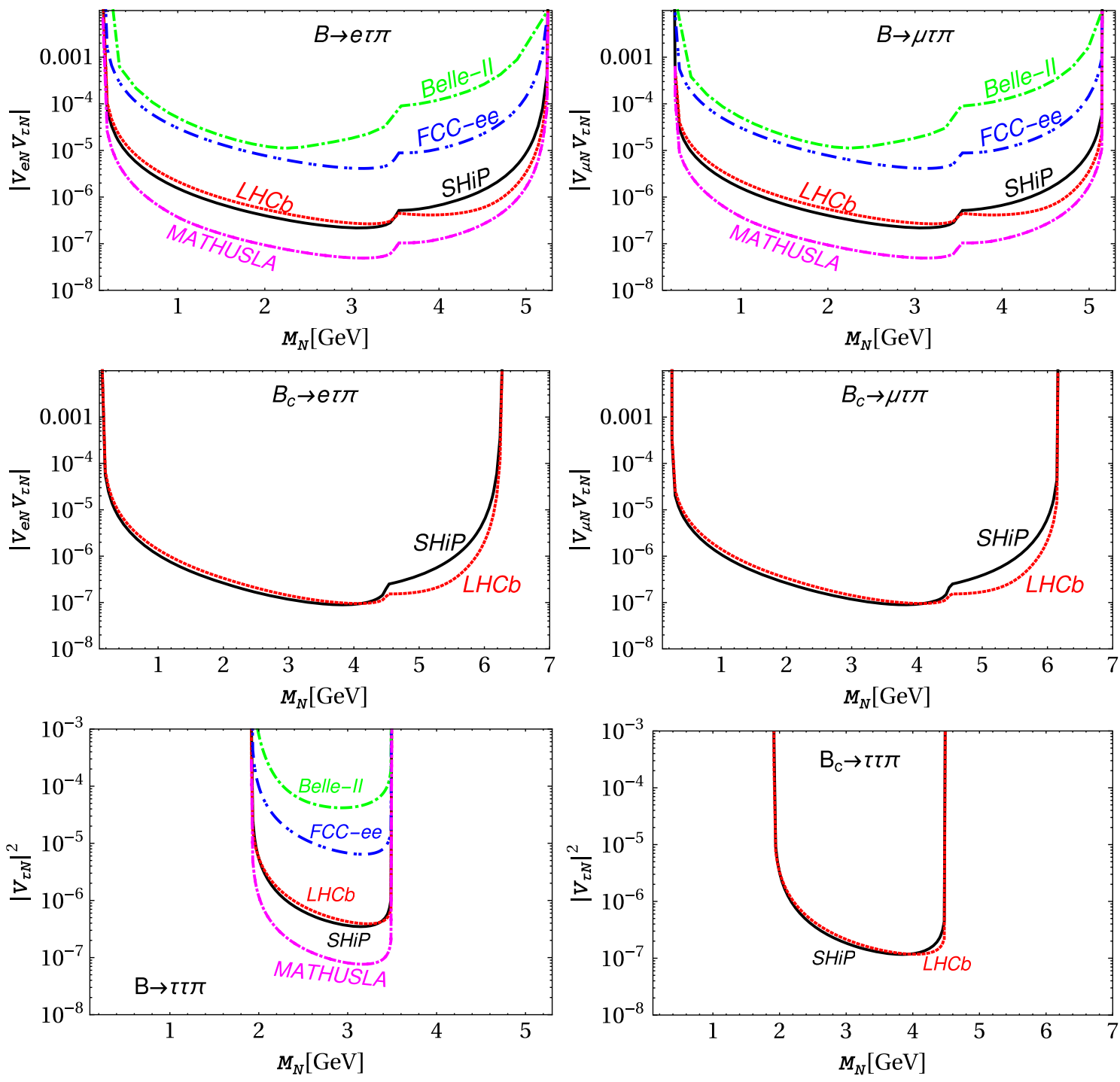

FIG. 10. Future sensitivity reach and present limits on the mixing angles $\left|V_{e N} V_{\tau N}\right|,\left|V_{\mu N} V_{\tau N}\right|$ and $\left|V_{\tau N}\right|^{2}$ with respect to the mass $M_{N}$ from meson decays $B, B_{c} \rightarrow e \tau \pi, B, B_{c} \rightarrow \mu \tau \pi$ and $B, B_{c} \rightarrow \tau \tau \pi$, respectively at various experiments. The upper panel is for $B$ meson decay at SHiP (black), MATHUSLA (magenta), LHCb (red), FCC-ee (blue) and Belle-II (green). The middle panel is for $B_{c}$ meson decay at SHiP (black) and LHCb (red). The left figure of the lower panel is for the bound on $\left|V_{\tau N}\right|^{2}$ from $B \rightarrow \tau \tau \pi$ decay at SHiP (black), MATHUSLA (magenta), LHCb (red), FCC-ee (blue) and Belle-II (green). The right figure of the lower panel is for the bound on $\left|V_{\tau N}\right|^{2}$ from $B_{c} \rightarrow \tau \tau \pi$ decay at SHiP (black) and $\mathrm{LHCb}$ (red).

obtained. The RH neutrino of higher mass $M_{N} \sim 5 \mathrm{GeV}$ and $M_{N} \sim 6 \mathrm{GeV}$ can be probed by $B \rightarrow e \mu \pi$ mode (by MATHUSLA) and $B_{c} \rightarrow e \mu \pi$ mode (by LHCb), with sensitivity reach $\left|V_{e N} V_{\mu N}\right| \sim 10^{-7}$. For $\left|V_{e N} V_{\tau N}\right|$ and $\left|V_{\mu N} V_{\tau N}\right|$ mixings, the sensitivity for the active-sterile mixing angles are similar, as depicted in Figs. 15 and 16. These can be probed in LHCb, MATHUSLA and SHiP. We note that the future limits from LNV meson decays will be most sensitive in between $0.5 \mathrm{GeV}<$ $M_{N}<2 \mathrm{GeV}$ for $\left|V_{e N}\right|^{2},\left|V_{\mu N}\right|^{2},\left|V_{e N} V_{\mu N}\right|$. For other mixing angles that involve $\tau$ in the final state, the best limit can be obtained in relatively higher mass range $M_{N} \sim 5 \mathrm{GeV}$. We stress that $\mathrm{LHCb}$ and future experiments
SHiP, MATHUSLA can probe mixing angle of the $\tau$ sector in a region that is very loosely constrained.

A number of other constraints on the active-sterile mixing have been obtained from peak searches, pion decays, collider searches, etc. A variety of choices of the heavy neutrino mass have been made in different articles [29,31,33,38,91-105], which discussed the limits on the heavy neutrino mass and mixing. We summarize the existing limits from these articles. We only show the constraints for the heavy neutrinos lighter than $10 \mathrm{GeV}$. See Fig. 11 for the two electron, Fig. 12 for two muon, and Fig. 13 for two tau final states, which constrain $\left|V_{e N}\right|^{2},\left|V_{\mu N}\right|^{2}$ and $\left|V_{\tau N}\right|^{2}$. These bounds in the mass vs 


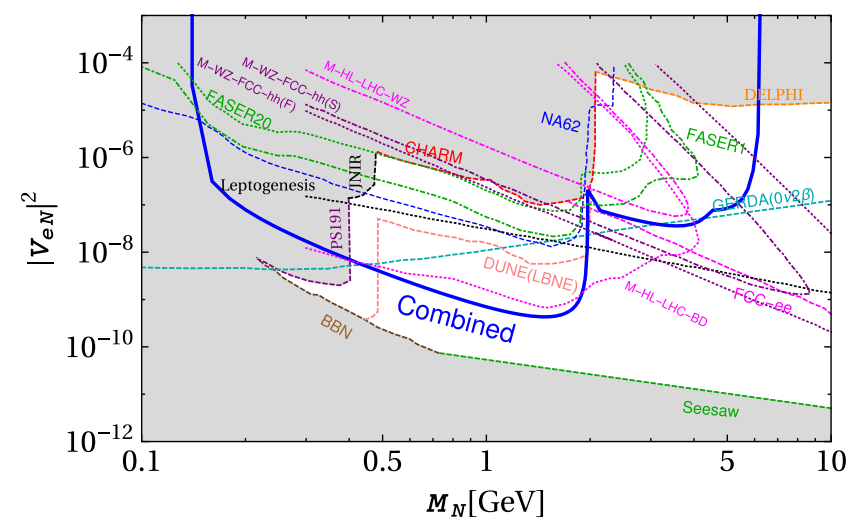

FIG. 11. Combined bounds (thick-blue solid) on mixing angle $\left|V_{e N}\right|^{2}$ as a function of mass $M_{N}$ from all the meson decays. The strongest lower limits from the Seesaw (green dashed) and BBN (brown dashed) on $\left|V_{e N}\right|^{2}$ are shown in this plot. The strongest upper bounds on $\left|V_{e N}\right|^{2}$ are obtained from PS191 (magenta dashed), JNIR (black dashed), CHARM (red dashed), DELPHI (orange dashed) and leptogenesis (black dotted) are shown in this plot. The shaded region is ruled out by these results. The projected upper limits from the NA62 (blue dashed), GERDA (dark-cyan dashed), FCC-ee (magenta dashed) and DUNE (pink dashed) on $\left|V_{e N}\right|^{2}$ are shown. Prospective bounds from the FASER with detector radius $R=20 \mathrm{~cm}$ is shown by FASER20 (green dotted) whereas the limits from the $R=1 \mathrm{~m}$ are shown by FASER1 (green-dot dashed). Prospective upper limits from the MATHUSLA at the FCC-hh for the $W / Z$ boson decays at the FCC-hh for MATHUSLA standard benchmark surface version are represented by M-WZ-FCC-hh(S) (magenta-dot dashed) and forward version M-WZ-FCC-hh(F) (magenta dotted). Prospective limits for the heavy neutrinos produced from the $W / Z$ decays are represented by M-HL-LHC-WZ (magenta-dot dashed) and $B / D$ meson decays are represented by M-HL-LHC-BD (magenta dotted) at the HL-LHC.

mixing plane of Figs. 11 to 13 represent the theory constraint from the seesaw (Seesaw) [106-108], big bang nucleosynthesis (BBN) $[69,109,110]$, experimental constraint from CHARM [111-113], and DELPHI [114]. We also show the future sensitivity reach of FCC-ee $[115,116]$ and DUNE (LBNE) [117]. The PS191 [118] limits for the electron and muon flavors are shown in Figs. 11 and 12, respectively. The JNIR [119] limit is represented by the black dashed line for the electron flavor in Fig. 11. The regions excluded by the present constraints are shaded in gray. The limits from GERDA [120] on the mass mixing plane in search of Majorana neutrinos from the neutrinoless double beta decay are represented by the dark cyan line in Fig. 11. Majorana heavy neutrino searches from the meson decay in E949 [121] and NuTeV [122] can produce strong bounds on the heavy neutrino mass-mixing plane. Leptonjet theoretical searches [123] for the Majorana neutrinos with muon flavor can also put strong bounds. These bounds are shown in Fig. 12 for the muon flavors. For the tau lepton, the bound in the corresponding mass region from

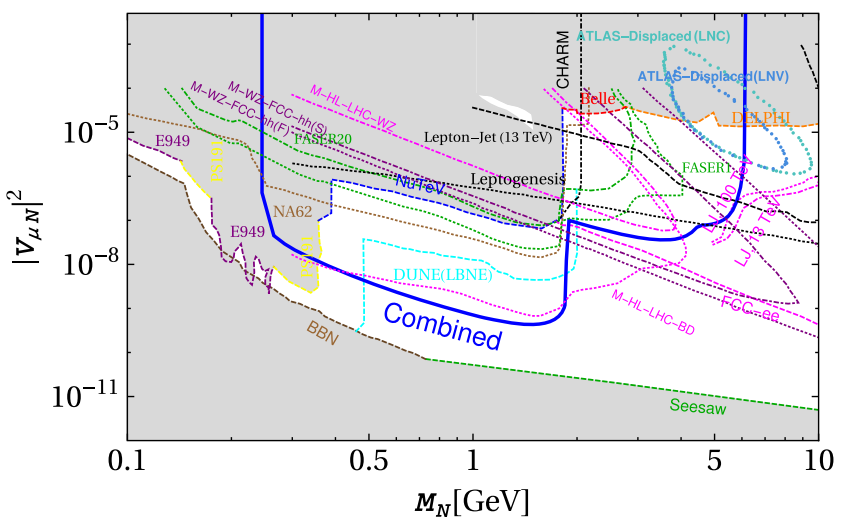

FIG. 12. Combined bounds (thick-blue solid) on mixing angle $\left|V_{\mu N}\right|^{2}$ as a function of mass $M_{N}$ from all the meson decays. Strongest lower limits from the seesaw (green dashed) and BBN (brown dashed) and strongest upper limits from PS191 (yellow dashed), E949 (magenta dashed), NuTeV (blue dashed), CHARM (black dashed), Belle (red dashed) and DELPHI (orange dashed) are shown in this plot. The shaded region is ruled out by these results. The projected upper limits from the NA62 (brown dotted), leptogenesis (black dotted), FCC-ee (magenta dashed) and DUNE (cyan dashed) and FASER with detector radius $R=20 \mathrm{~cm}$ is shown by FASER20 (green-dot dashed) whereas the limits from the $R=1 \mathrm{~m}$ are shown by FASER1 (green dotted). Prospective upper limits from the MATHUSLA at the FCC-hh for the $W / Z$ boson decays at the FCC-hh for MATHUSLA standard benchmark surface version are represented by M-WZ-FCC-hh(S) (magenta-dot dashed) and forward version M-WZ-FCC-hh(F) (magenta dotted). Prospective limits for the heavy neutrinos produced from the $W / Z$ decays are represented by M-HL-LHC-WZ (magenta-dot dashed) and $B / D$ meson decays are represented by M-HL-LHC-BD (magenta dotted) at the HL-LHC. Experimental bounds from the ATLAS displaced vertex searches of the Majorana heavy neutrino for the lepton number violating (LNV) channel are represented by ATLAS-displaced (LNV) (light-blue dotted) and the limits obtained from the lepton number conserving (LNC) channel are represented by ATLAS-displaced (LNC) (sea-blue dotted). Theoretical limits from lepton-jet search for $1 \mathrm{GeV} \leq M_{N} \leq$ $10 \mathrm{GeV}$ has been represented by lepton jet (13 TeV) (black dashed). Corresponding limits for $M_{N} \geq 5 \mathrm{GeV}$ for the $13 \mathrm{TeV}$ LHC and $100 \mathrm{TeV}$ are shown by LJ, $13 \mathrm{TeV}$ (magenta-dot dashed) and LJ, $100 \mathrm{TeV}$ (magenta dotted) respectively.

EWPD [124-126] has been shown in Fig. 13. The decay of tau lepton into heavy Majorana neutrino and meson can also put bounds on the mass-mixing plane and can have prospective limits marked as B-factory $[92,95] .^{2}$ The NA62 [128-130] projection lines from the electron, muon and tau are shown in Figs. 11, 12, and 13, respectively. Such an experiment can be performed in the kaon mode and beam dump mode [131]. This search is sensitive to the heavy neutrinos that are produced in weak decays

\footnotetext{
${ }^{2}$ Recently, Ref [127] also put bound on $\left|V_{\tau N}\right|^{2}$ using large samples of $e^{+} e^{-} \rightarrow \tau^{+} \tau^{-}$collected at B-factory experiments.
} 


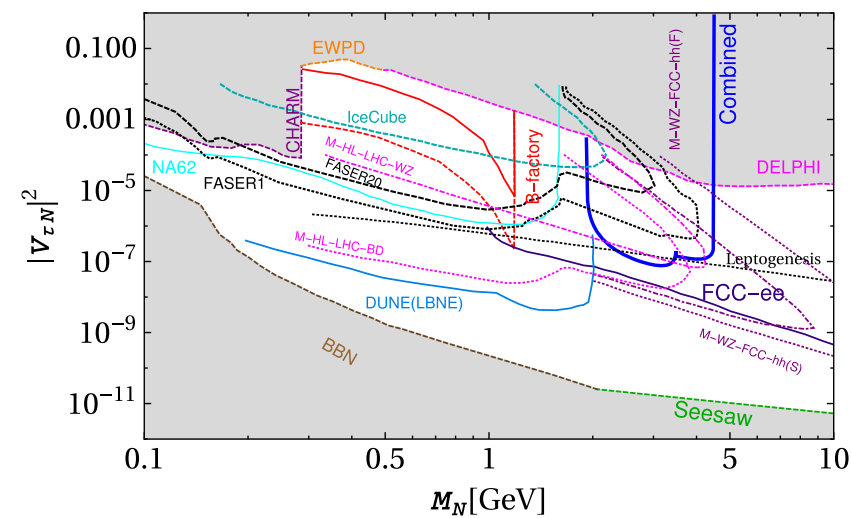

FIG. 13. Combined bounds (thick-blue solid) on mixing angle $\left|V_{\tau N}\right|^{2}$ as a function of mass $M_{N}$ from all the meson decays. Strongest lower limits from the Seesaw (green dashed) and BBN (brown dashed) on $\left|V_{\tau N}\right|^{2}$ are shown in this plot. The strongest upper bounds on $\left|V_{\tau N}\right|^{2}$ are obtained from CHARM (purple dashed), EWPD (orange dashed), leptogenesis (black dotted), DELPHI (magenta dashed) are shown in this plot. The shaded region is ruled out by these results. The projected upper limits from the NA62 (cyan solid), FCC-ee (darker-blue solid) and DUNE (light-blue solid) on $\left|V_{\tau N}\right|^{2}$ are shown. Prospective bounds from the FASER with detector radius $R=20 \mathrm{~cm}$ is shown by FASER20 (black dashed) whereas the limits from the $R=1 \mathrm{~m}$ is shown by FASER1 (black dotted). Prospective upper limits from the MATHUSLA at the FCC-hh for the $W / Z$ boson decays at the FCC-hh for MATHUSLA standard benchmark surface version are represented by M-WZ-FCC-hh(S) (magentadot dashed) and forward version M-WZ-FCC-hh(F) (magenta dotted). Prospective limits for the heavy neutrinos produced from the $W / Z$ decays are represented by M-HL-LHC-WZ (magentadot dashed) and $B / D$ meson decays are represented by M-HLLHC-BD (magenta dotted) at the HL-LHC. The prospective limits from the B-factory (red dashed and red solid) and IceCube (darker-cyan dashed) are also shown in this figure.

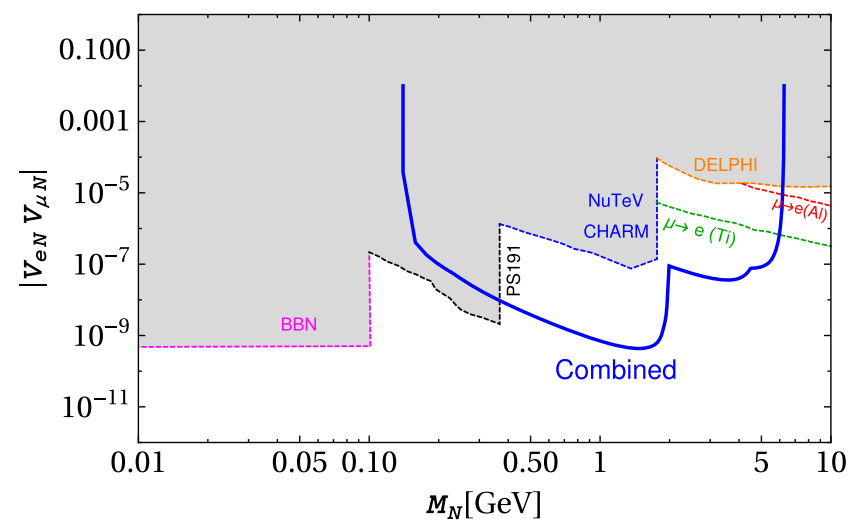

FIG. 14. Combined bounds on mixing angle $\left|V_{e N} V_{\mu N}\right|$ as a function of mass $M_{N}$ from all the meson decays. The other strongest bounds on $\left|V_{e N} V_{\mu N}\right|$ from BBN (magenta dashed), PS191 (black dashed), NuTeV, CHARM (blue dashed), DELPHI (orange dashed) are also shown in this figure. Prospective bounds on $\left|V_{e N} V_{\mu N}\right|$ from the $\mu \rightarrow e$ (Ti) (green dashed) and $\mu \rightarrow e$ (Al) (red dashed) are also shown for $M_{N} \leq 10 \mathrm{GeV}$.

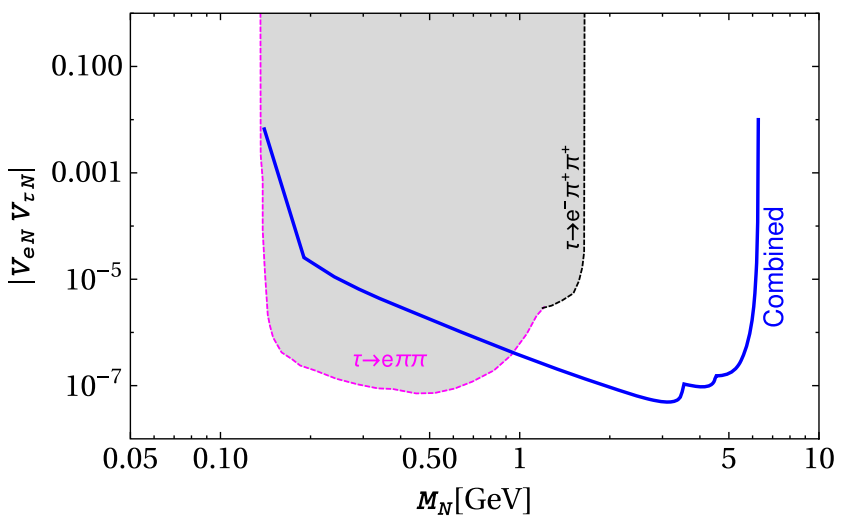

FIG. 15. Combined bounds (thick-blue solid) on mixing angle $\left|V_{e N} V_{\tau N}\right|$ as a function of mass $M_{N}$ from all the meson decays. Upper limits from $\tau \rightarrow e \pi \pi$ (magenta dashed) and $\tau \rightarrow e^{-} \pi^{+} \pi^{+}$ (black dashed) are also shown for $M_{N} \leq 10 \mathrm{GeV}$.

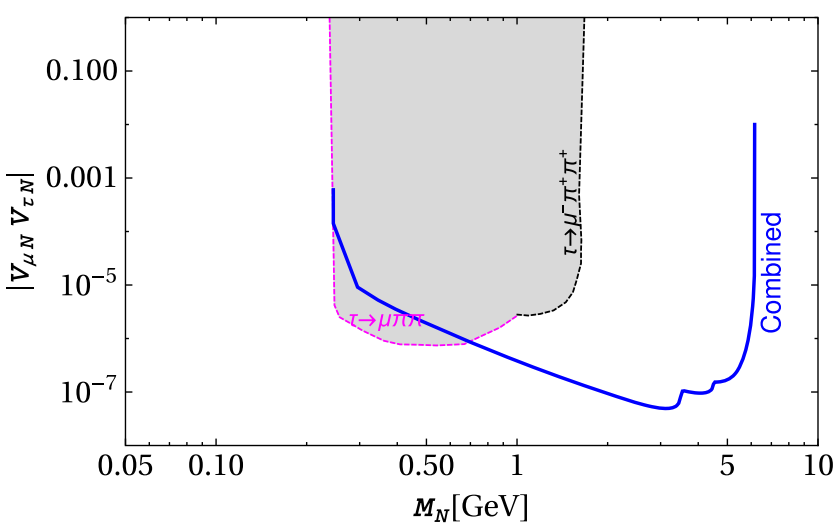

FIG. 16. Combined bounds (thick-blue solid) on mixing angle $\left|V_{\mu N} V_{\tau N}\right|$ as a function of mass $M_{N}$ from all the meson decays. Upper limits from $\tau \rightarrow \mu \pi \pi$ (magenta dashed) and $\tau \rightarrow \mu^{-} \pi^{+} \pi^{+}$ (black dashed) are also shown for $M_{N} \leq 10 \mathrm{GeV}$.

$[132,133]$ of mesons or tau leptons [69]. The upper limit on the mass mixing plane from the leptogenesis [134] for the minimal scenario with two right-handed neutrinos are shown in Figs. 11 and 12, respectively for the electrons and muons assuming the normal hierarchy of the light neutrino masses. The upper limit on the mixing angle $\left|V_{\mu N}\right|^{2}$ from Belle [88] are shown in Fig. 12. Projected sensitivities (for the four events) of the MATHUSLA [83] detector in the mass-mixing plane for the heavy neutrinos produced from the $W / Z$ decays at the FCC-hh for MATHUSLA standard benchmark surface version (M-WZ-FCC-hh(S)) and forward version (M-WZ-FCC-hh(F)) for the electron, muon and tau are shown in Figs. 11, 12 and 13, respectively for $M_{N}>2 \mathrm{GeV}$. We have shown the projected sensitivities in the mass-mixing plane for the heavy neutrinos produced from the $W / Z$ decays (M-HL-LHC-WZ) and $B / D$ meson decays (M-HL-LHC-BD) for electron, muon and tau lepton 
at the HL-LHC in Figs. 11, 12 and 13, respectively. The bounds on the mass-mixing plane from the FASER [135] with detector radius $R=20 \mathrm{~cm}$ have been represented by FASER 20 and $R=1 \mathrm{~m}$ has been represented by FASER 1 , respectively for the electron, muon and tau leptons in Figs. 11, 12 and 13, respectively. ATLAS displaced vertex bounds on the $\left|V_{\mu N}\right|^{2}$ for the lepton number violating (LNV) and lepton number conserving (LNC) searches are given in 12 [136]. The prospective upper bounds in the mass-mixing plane for the tau lepton from the IceCube [137-140] for $M_{N}<10 \mathrm{GeV}$ are shown in Fig. 13. The projected sensitivity (theoretical) on $\left|V_{\mu N}\right|^{2}$ for the lepton-jet search for the for $1 \mathrm{GeV} \leq M_{N} \leq 10 \mathrm{GeV}$ at the $13 \mathrm{TeV}$ are shown in Fig. 12, by the black dashed line, and the line labeled by Lepton-Jet (13 TeV).The corresponding limits (theoretical) on $\left|V_{\mu N}\right|^{2}$ from another lepton-jet search [123] for $M_{N} \geq$ $5 \mathrm{GeV}$ at $13 \mathrm{TeV}$ (LJ, $13 \mathrm{TeV}$ ) and $100 \mathrm{TeV}(\mathrm{LJ}, 100 \mathrm{TeV})$ are also shown in Fig. 12.

We briefly summarize the current strongest experimental bounds on the mixing angles such as $\left|V_{e N} V_{\mu N}\right|,\left|V_{e N} V_{\tau N}\right|$, and $\left|V_{\mu N} V_{\tau N}\right|$ for the Majorana heavy neutrinos in Figs. 14,15 and 16 , respectively for $M_{N}<10 \mathrm{GeV}$. Strongest bounds from the BBN $[69,109,110]$, PS191 [118], NuTeV [122], CHARM [111-113], and DELPHI [114] are obtained from the Majorana heavy neutrino search for $M_{N} \leq 10 \mathrm{GeV}$. The shaded region is excluded by the results obtained from these experiments. The prospective bounds from the $\mu \rightarrow e$ (Ti) and $\mu \rightarrow e$ (Al) are shown in Fig. 14 from [141]. The limits on the mixings from the $\tau$ decay into hadrons in association with electron and muon are shown in Figs. 15 and 16, respectively from BABAR [142]. The limits from the $\tau \rightarrow e \pi \pi$ and $\tau \rightarrow e^{-} \pi^{+} \pi^{+}$are shown in Fig. 15 and those obtained from $\tau \rightarrow \mu \pi \pi$ and $\tau \rightarrow \mu^{-} \pi^{+} \pi^{+}$are shown in Fig. 16, respectively [143].

We stress that, in the relatively lower mass range, among the experimental constraints, the tightest constraint on the mixing angles $\left|V_{e N}\right|^{2}$ and $\left|V_{\mu N}\right|^{2}$ can be obtained from LNV meson decays. These are however still 1 order of magnitude weaker than the theory constraints from BBN and Seesaw. For relatively higher mass range, our combined bounds on the mixing angles $\left|V_{e N} V_{\mu N}\right|,\left|V_{e N} V_{\tau N}\right|$ and $\left|V_{\mu N} V_{\tau N}\right|$ are the tightest bounds. As we have discussed before, the LNV meson decays can probe the product of the mixings $\left|V_{\mu N} V_{\tau N}\right|,\left|V_{e N} V_{\tau N}\right|$ in higher mass ranges $M_{N} \sim 5 \mathrm{GeV}$, which are so far unconstrained.

\section{CONCLUSION}

We analyze the discovery prospect of a heavy Majorana neutrino via lepton number violating meson decays $M_{1}^{-} \rightarrow$ $\ell_{1}^{-} \ell_{2}^{-} \pi^{+}$at various ongoing and future experiments, such as NA62, LHCb, FCC-ee, Belle-II, SHiP and MATHUSLA. The large number of decaying mesons in these experiments may possibly result in an observation of the different rare lepton number violating decays. Even their nonobservation can be used to set constraints on the mixing coefficients between the standard flavor neutrinos and the heavy mass eigenstates. We explore in detail the effect of parent meson's velocity on the sensitivity reach of the activesterile mixing angles in the ongoing and future experiments. We compare the resulting constraints on the mixing angles for the case of meson decay at rest with that of meson decaying in flight, with the former being much tighter. We stress that significant difference in the mixing angles can occur for experiments NA62, LHCb, and the future experiment SHiP. Due to nonzero velocity of the parent meson, the probability of the generated $\mathrm{RH}$ neutrino to decay inside the detector changes. This alters the sensitivity reach by more than an order of magnitude for the above-mentioned experiments.

We explore a number of channels, $B / D / D_{s} \rightarrow \mu \mu \pi$, $B / D / D_{s} \rightarrow e e \pi, B_{c} / D_{s} \rightarrow e \mu \pi, B \rightarrow \tau \tau \pi, B / B_{c} \rightarrow e \tau \pi$, $B / B_{c} \rightarrow \mu \tau \pi$, and few others. We find that, for the mass range $M_{N} \sim 1 \mathrm{GeV}$, future experiment SHiP can probe $\left|V_{e N}\right|^{2} \sim 10^{-9}$, while for mass range $M_{N} \sim 5 \mathrm{GeV}$, future experiment MATHUSLA, and LHCb with $300 \mathrm{fb}^{-1}$ integrated luminosity can probe $\left|V_{e N}\right|^{2} \sim 10^{-7}$. The sensitivity reach of $\left|V_{\mu N}\right|^{2}$ is very similar to $\left|V_{e N}\right|^{2}$. For $\left|V_{\tau N}\right|^{2}$, the best sensitivity reach $\left|V_{\tau N}\right|^{2} \sim 10^{-7}$ can be provided by MATHUSLA in the mass range $2 \mathrm{GeV}<M_{N}<3.4 \mathrm{GeV}$, while in the mass range $3.4 \mathrm{GeV}<M_{N}<4.4 \mathrm{GeV}$, SHiP and LHCb give similar sensitivity reach. For $\left|V_{e N} V_{\mu N}\right|$, mass range up to $M_{N} \sim 2 \mathrm{GeV}$ can be probed at SHiP with the sensitivity reach $\left|V_{e N} V_{\mu N}\right| \sim 10^{-9}$, while higher mass $M_{N} \sim 5 \mathrm{GeV}$ and $M_{N} \sim 6 \mathrm{GeV}$ can be probed at MATHUSLA and LHCb with sensitivity reach $\left|V_{e N} V_{\mu N}\right| \sim$ $10^{-7}$. The highest sensitivity reach on $\left|V_{e N} V_{\tau N}\right|,\left|V_{\mu N} V_{\tau N}\right| \sim$ $10^{-7}$ can be provided by MATHUSLA and LHCb. The combined sensitivity of the mixing angles $\left|V_{e N} V_{\mu N}\right|$, $\left|V_{e N} V_{\tau N}\right|$ and $\left|V_{\mu N} V_{\tau N}\right|$ from meson decays is tighter than the other constraints available in a large range of heavy neutrino mass.

\section{ACKNOWLEDGMENTS}

The work of A. D. is supported by the Japan Society for the Promotion of Science (JSPS) Post-doctoral Fellowship for Research in Japan. M. M. acknowledges the support of DST INSPIRE Research Grant No. IFA14-PH-99.

\section{APPENDIX}

The different partial decay widths of the RH neutrinos $N_{i}$ are

$$
\Gamma\left(N_{j} \rightarrow \ell^{-} P^{+}\right)=\frac{G_{F}^{2} M_{N_{j}}^{3}}{16 \pi} f_{p}^{2}\left|V_{q \bar{q}^{\prime}}^{\mathrm{CKM}}\right|^{2}\left|V_{\ell_{1} N_{j}}\right|^{2} F_{P}\left(x_{\ell}, x_{P}\right),
$$




$$
\begin{aligned}
& \Gamma\left(N_{j} \rightarrow \ell^{-} V^{+}\right)=\frac{G_{F}^{2} M_{N_{j}}^{3}}{16 \pi} f_{V}^{2}\left|V_{q \bar{q}^{\prime}}^{\mathrm{CKM}}\right|^{2}\left|V_{\ell_{1} N_{j}}\right|^{2} F_{V}\left(x_{\ell}, x_{V}\right) \\
& \Gamma\left(N_{j} \rightarrow \nu_{\ell} P^{0}\right)=\frac{G_{F}^{2} M_{N_{j}}^{3}}{4 \pi} f_{P}^{2} \sum_{i}\left|U_{\ell i}\right|^{2}\left|V_{\ell_{1} N_{j}}\right|^{2} K_{P}^{2} F_{P}\left(x_{\nu_{\ell}}, x_{P}\right), \\
& \Gamma\left(N_{j} \rightarrow \nu_{\ell} V^{0}\right)=\frac{G_{F}^{2} M_{N_{j}}^{3}}{4 \pi} f_{V}^{2} \sum_{i}\left|U_{\ell i}\right|^{2}\left|V_{\ell_{1} N_{j}}\right|^{2} K_{V}^{2} F_{V}\left(x_{\nu_{\ell}}, x_{P}\right), \\
& \Gamma\left(N_{j} \rightarrow \ell_{1}^{-} \ell_{2}^{+} \nu_{\ell_{2}}\right)=\frac{G_{F}^{2} M_{N_{j}}^{5}}{16 \pi^{3}}\left|V_{\ell_{1} N_{j}}\right|^{2} \sum_{i}\left|U_{\ell_{2} i}\right|^{2} I_{1}\left(x_{\ell_{1}}, x_{\nu_{\ell_{2}}}, x_{\ell_{2}}\right), \\
& \Gamma\left(N_{j} \rightarrow \nu_{\ell_{2}} \ell_{2}^{-} \ell_{2}^{+}\right)=\frac{G_{F}^{2} M_{N_{j}}^{5}}{16 \pi^{3}}\left|V_{\ell_{2} N_{j}}\right|^{2} \sum_{i}\left|U_{\ell_{2} i}\right|^{2}\left[I_{1}\left(x_{\nu_{\ell_{2}}}, x_{\ell_{2}}, x_{\ell_{2}}\right)+2\left(\left(g_{V}^{\ell}\right)^{2}+\left(g_{A}^{\ell}\right)^{2}\right)\right. \\
& \left.\times I_{1}\left(x_{\nu_{\ell_{2}}}, x_{\ell_{2}}, x_{\ell_{2}}\right)+2\left(\left(g_{V}^{\ell}\right)^{2}-\left(g_{A}^{\ell}\right)^{2}\right) I_{2}\left(x_{\nu_{\ell_{2}}}, x_{\ell_{2}}, x_{\ell_{2}}\right)\right] \\
& \Gamma\left(N_{j} \rightarrow \nu_{\ell_{1}} \ell_{2}^{-} \ell_{2}^{+}\right)=\frac{G_{F}^{2} M_{N_{j}}^{5}}{8 \pi^{3}}\left|V_{\ell_{1} N_{j}}\right|^{2} \sum_{i}\left|U_{\ell_{1} i}\right|^{2}\left[\left(\left(g_{V}^{\ell}\right)^{2}+\left(g_{A}^{\ell}\right)^{2}\right) I_{1}\left(x_{\nu_{\ell_{1}}}, x_{\ell_{2}}, x_{\ell_{2}}\right)+\left(\left(g_{V}^{\ell}\right)^{2}-\left(g_{A}^{\ell}\right)^{2}\right) I_{2}\left(x_{\nu_{\ell_{1}}}, x_{\ell_{2}}, x_{\ell_{2}}\right)\right]
\end{aligned}
$$

In the above decay mode $\ell_{1} \neq \ell_{2}$ :

$$
\Gamma\left(N_{j} \rightarrow \nu_{\ell} \nu \bar{\nu}\right)=\frac{G_{F}^{2} M_{N_{j}}^{5}}{192 \pi^{3}}\left|V_{\ell N_{j}}\right|^{2} \sum_{i}\left|U_{\ell i}\right|^{2}
$$

where $x_{i}=\frac{m_{i}}{M_{N}}$ with $m_{i}=m_{\ell}, m_{P^{0}}, m_{V^{0}}, m_{P^{+}}, m_{V}^{+}$. The kinematical functions are given by

$$
\begin{aligned}
I_{1}(x, y, z) & =\int_{(x+y)^{2}}^{(1-z)^{2}} \frac{d s}{s}\left(s-x^{2}-y^{2}\right)\left(1+z^{2}-s\right) \lambda^{\frac{1}{2}}\left(s, x^{2}, y^{2}\right) \lambda^{\frac{1}{2}}\left(1, s, z^{2}\right) \\
I_{2}(x, y, z) & =y z \int_{(y+z)^{2}}^{(1-x)^{2}} \frac{d s}{s}\left(1+x^{2}-s\right) \lambda^{\frac{1}{2}}\left(s, y^{2}, z^{2}\right) \lambda^{\frac{1}{2}}\left(1, s, x^{2}\right) \\
F_{P}(x, y) & =\left(\left(1+x^{2}\right)\left(1+x^{2}-y^{2}\right)-4 x^{2}\right) \lambda^{\frac{1}{2}}\left(1, x^{2}, y^{2}\right) \\
F_{V}(x, y) & =\left(\left(1-x^{2}\right)^{2}+\left(1+x^{2}\right) y^{2}-2 y^{4}\right) \lambda^{\frac{1}{2}}\left(1, x^{2}, y^{2}\right) .
\end{aligned}
$$

Neutral current couplings of leptons are given by

$$
g_{V}^{\ell}=-\frac{1}{4}+\sin ^{2} \theta_{w}, \quad g_{A}^{\ell}=\frac{1}{4} .
$$

Neutral current coupling of pseudoscalar and vector mesons is given by

$$
\begin{aligned}
& K_{\pi^{0}}=-\frac{1}{2 \sqrt{2}}, \quad K_{\eta}=-\frac{1}{2 \sqrt{6}}, \quad K_{\eta^{\prime}}=\frac{1}{4 \sqrt{3}}, \quad K_{\eta_{c}}=-\frac{1}{4}, \quad K_{\rho^{0}}=\frac{1}{\sqrt{2}}\left(\frac{1}{2}-\sin ^{2} \theta_{w}\right), \\
& K_{\omega}=-\frac{1}{3 \sqrt{2}} \sin ^{2} \theta_{w}, \quad K_{\phi}=\left(-\frac{1}{4}+\frac{1}{3} \sin ^{2} \theta_{w}\right), \quad K_{J / \psi}=\left(\frac{1}{4}-\frac{2}{3} \sin ^{2} \theta_{w}\right) .
\end{aligned}
$$


[1] M. C. Gonzalez-Garcia and M. Maltoni, Phenomenology with massive neutrinos, Phys. Rep. 460, 1 (2008).

[2] M. Lattanzi and M. Gerbino, Status of neutrino properties and future prospects-Cosmological and astrophysical constraints, Front. Phys. 5, 70 (2018).

[3] S. Weinberg, Baryon and Lepton Nonconserving Processes, Phys. Rev. Lett. 43, 1566 (1979).

[4] P. Minkowski, $\mu \rightarrow e \gamma$ at a rate of one out of $10^{9}$ muon decays?, Phys. Lett. 67B, 421 (1977).

[5] T. Yanagida, in Proceedings of the Workshop on Grand Unified Theory and Baryon Number of the Universe, KEK, Japan, 1979, edited by O. Sawada and A. Sugamoto (1979).

[6] P. Ramond, The family group in grand unified theories, in International Symposium on Fundamentals of Quantum Theory and Quantum Field Theory Palm Coast, Florida, 1979 (1979), pp. 265-280.

[7] R. N. Mohapatra and G. Senjanovic, Neutrino Mass and Spontaneous Parity Nonconservation, Phys. Rev. Lett. 44, 912 (1980).

[8] R. N. Mohapatra, Mechanism for Understanding Small Neutrino Mass in Superstring Theories, Phys. Rev. Lett. 56, 561 (1986).

[9] R. N. Mohapatra and J. W. F. Valle, Neutrino mass and baryon number nonconservation in superstring models, Phys. Rev. D 34, 1642 (1986).

[10] M. Magg and C. Wetterich, Neutrino mass problem and gauge hierarchy, Phys. Lett. 94B, 61 (1980).

[11] T. P. Cheng and L.-F. Li, Neutrino masses, mixings and oscillations in $\mathrm{SU}(2) \times \mathrm{U}(1)$ models of electroweak interactions, Phys. Rev. D 22, 2860 (1980).

[12] G. Lazarides, Q. Shafi, and C. Wetterich, Proton lifetime and fermion masses in an SO(10) model, Nucl. Phys. B181, 287 (1981).

[13] R. N. Mohapatra and G. Senjanovic, Neutrino masses and mixings in gauge models with spontaneous parity violation, Phys. Rev. D 23, 165 (1981).

[14] R. Foot, H. Lew, X. G. He, and G. C. Joshi, Seesaw neutrino masses induced by a triplet of leptons, Z. Phys. C 44, 441 (1989).

[15] E. Ma, Pathways to Naturally Small Neutrino Masses, Phys. Rev. Lett. 81, 1171 (1998).

[16] B. Bajc and G. Senjanovic, Seesaw at LHC, J. High Energy Phys. 08 (2007) 014.

[17] P. Fileviez Perez, Renormalizable adjoint SU(5), Phys. Lett. B 654, 189 (2007).

[18] W.-Y. Keung and G. Senjanovic, Majorana Neutrinos and the Production of the Right-Handed Charged Gauge Boson, Phys. Rev. Lett. 50, 1427 (1983).

[19] A. M. Sirunyan et al. (CMS Collaboration), Search for heavy Majorana neutrinos in same-sign dilepton channels in proton-proton collisions at $\sqrt{s}=13 \mathrm{TeV}$, J. High Energy Phys. 01 (2019) 122.

[20] G. Aad et al. (ATLAS Collaboration), Search for doublycharged Higgs bosons in same-charge electron pair final states using proton-proton collisions at $\sqrt{s}=13 \mathrm{TeV}$ with the ATLAS detector, Technical Report No. ATLASCONF-2016-051, CERN, Geneva, 2016, http://cds.cern .ch/record/2206133.
[21] F. del Aguila and J. A. Aguilar-Saavedra, Distinguishing seesaw models at LHC with multi-lepton signals, Nucl. Phys. B813, 22 (2009).

[22] A. Das and N. Okada, Bounds on heavy Majorana neutrinos in type-I seesaw and implications for collider searches, Phys. Lett. B 774, 32 (2017).

[23] A. Das, P. Konar, and A. Thalapillil, Jet substructure shedding light on heavy Majorana neutrinos at the LHC, J. High Energy Phys. 02 (2018) 083.

[24] F. del Aguila and J. A. Aguilar-Saavedra, Electroweak scale seesaw and heavy Dirac neutrino signals at LHC, Phys. Lett. B 672, 158 (2009).

[25] S. Pascoli, R. Ruiz, and C. Weiland, Heavy neutrinos with dynamic jet vetoes: Multilepton searches at $\sqrt{s}=14,27$, and $100 \mathrm{TeV}$, J. High Energy Phys. 06 (2019) 049.

[26] A. Das and N. Okada, Improved bounds on the heavy neutrino productions at the LHC, Phys. Rev. D 93, 033003 (2016).

[27] A. Das, P. Konar, and S. Majhi, Production of heavy neutrino in next-to-leading order QCD at the LHC and beyond, J. High Energy Phys. 06 (2016) 019.

[28] A. M. Sirunyan et al. (CMS Collaboration), Search for Heavy Neutral Leptons in Events with Three Charged Leptons in Proton-Proton Collisions at $\sqrt{s}=13 \mathrm{TeV}$, Phys. Rev. Lett. 120, 221801 (2018).

[29] D. Das, K. Ghosh, M. Mitra, and S. Mondal, Probing sterile neutrinos in the framework of inverse seesaw mechanism through leptoquark productions, Phys. Rev. D 97, 015024 (2018).

[30] G. Cottin, J. C. Helo, and M. Hirsch, Displaced vertices as probes of sterile neutrino mixing at the LHC, Phys. Rev. D 98, 035012 (2018).

[31] S. Banerjee, P. S. B. Dev, A. Ibarra, T. Mandal, and M. Mitra, Prospects of heavy neutrino searches at future lepton colliders, Phys. Rev. D 92, 075002 (2015).

[32] S. Mondal and S. K. Rai, Probing the heavy neutrinos of inverse seesaw model at the LHeC, Phys. Rev. D 94, 033008 (2016).

[33] S. Antusch, E. Cazzato, and O. Fischer, Sterile neutrino searches at future $e^{-} e^{+}, p p$, and $e^{-} p$ colliders, Int. J. Mod. Phys. A 32, 1750078 (2017).

[34] S. Chakraborty, M. Mitra, and S. Shil, Fat jet signature of a heavy neutrino at lepton collider, Phys. Rev. D 100, 015012 (2019).

[35] S. Mandal, M. Mitra, and N. Sinha, Probing leptoquarks and heavy neutrinos at the LHeC, Phys. Rev. D 98, 095004 (2018).

[36] A. Das, S. Jana, S. Mandal, and S. Nandi, Probing right handed neutrinos at the $\mathrm{LHeC}$ and lepton colliders using fat jet signatures, Phys. Rev. D 99, 055030 (2019).

[37] R. Ruiz, M. Spannowsky, and P. Waite, Heavy neutrinos from gluon fusion, Phys. Rev. D 96, 055042 (2017).

[38] Y. Cai, T. Han, T. Li, and R. Ruiz, Lepton number violation: Seesaw models and their collider tests, Front. Phys. 6, 40 (2018).

[39] A. Bhardwaj, A. Das, P. Konar, and A. Thalapillil, Looking for minimal inverse seesaw scenarios at the LHC with jet substructure techniques, arXiv:1801.00797. 
[40] E. Izaguirre and B. Shuve, Multilepton and lepton jet probes of sub-weak-scale right-handed neutrinos, Phys. Rev. D 91, 093010 (2015).

[41] F. T. Avignone, III, S. R. Elliott, and J. Engel, Double beta decay, Majorana neutrinos, and neutrino mass, Rev. Mod. Phys. 80, 481 (2008).

[42] H. V. Klapdor-Kleingrothaus et al., Latest results from the Heidelberg-Moscow double beta decay experiment, Eur. Phys. J. A 12, 147 (2001).

[43] P. Benes, A. Faessler, F. Simkovic, and S. Kovalenko, Sterile neutrinos in neutrinoless double beta decay, Phys. Rev. D 71, 077901 (2005).

[44] M. Mitra, G. Senjanovic, and F. Vissani, Neutrinoless double beta decay and heavy sterile neutrinos, Nucl. Phys. B856, 26 (2012).

[45] S. Pascoli, M. Mitra, and S. Wong, Effect of cancellation in neutrinoless double beta decay, Phys. Rev. D 90, 093005 (2014).

[46] E. Ma and A. Pramudita, Exact formula for $(\mu \rightarrow e \gamma)$ type processes in the standard model, Phys. Rev. D 24, 1410 (1981).

[47] M. Duerr, D. P. George, and K. L. McDonald, Neutrino mass and $\mu \rightarrow e+\gamma$ from a mini-seesaw, J. High Energy Phys. 07 (2011) 103.

[48] C. A. Heusch and P. Minkowski, Lepton flavor violation induced by heavy Majorana neutrinos, Nucl. Phys. B416, 3 (1994).

[49] R. E. Shrock, New tests for, and bounds on, neutrino masses and lepton mixing, Phys. Lett. 96B, 159 (1980).

[50] A. Atre, T. Han, S. Pascoli, and B. Zhang, The search for heavy Majorana neutrinos, J. High Energy Phys. 05 (2009) 030 .

[51] L. S. Littenberg and R. Shrock, Implications of improved upper bounds on - Delta $L-=2$ processes, Phys. Lett. B 491, 285 (2000).

[52] L.S. Littenberg and R. E. Shrock, Upper Bounds on Lepton Number Violating Meson Decays, Phys. Rev. Lett. 68, 443 (1992).

[53] J. C. Helo, S. Kovalenko, and I. Schmidt, Sterile neutrinos in lepton number and lepton flavor violating decays, Nucl. Phys. B853, 80 (2011).

[54] G. Cvetic, C. Dib, S. K. Kang, and C. S. Kim, Probing Majorana neutrinos in rare $K$ and $D, D_{s}, \mathrm{~B}, B_{c}$ meson decays, Phys. Rev. D 82, 053010 (2010).

[55] G. Cvetic and C.S. Kim, Sensitivity bounds on heavy neutrino mixing $\left|U_{\mu N}\right|^{2}$ and $\left|U_{\tau N}\right|^{2}$ from LHCb upgrade, Phys. Rev. D 100, 015014 (2019).

[56] D. Milanés and N. Quintero, Search for lepton-numberviolating signals in the charm sector, Phys. Rev. D 98, 096004 (2018).

[57] H.-L. Li, P.-C. Lu, C.-F. Qiao, Z.-G. Si, and Y. Wang, Study standard model and Majorana neutrino contributions to $B^{+} \rightarrow K^{(*) \pm} \mu^{+} \mu^{\mp}$, Chin. Phys. C 43, 023101 (2019).

[58] A. Abada, V. De Romeri, M. Lucente, A. M. Teixeira, and T. Toma, Effective Majorana mass matrix from tau and pseudoscalar meson lepton number violating decays, J. High Energy Phys. 02 (2018) 169.

[59] H. Yuan, T. Wang, Y. Jiang, Q. Li, and G.-L. Wang, Fourbody decays of $B$ meson with lepton number violation, J. Phys. G 45, 065002 (2018).
[60] J. Mejia-Guisao, D. Milanés, N. Quintero, and J. D. RuizAlvarez, Lepton number violation in $B_{s}$ meson decays induced by an on-shell Majorana neutrino, Phys. Rev. D 97, 075018 (2018).

[61] G. Cvetic and C. S. Kim, Sensitivity limits on heavy-light mixing $\left|U_{\mu N}\right|^{2}$ from lepton number violating $B$ meson decays, Phys. Rev. D 96, 035025 (2017).

[62] S. Mandal, M. Mitra, and N. Sinha, Constraining the righthanded gauge boson mass from lepton number violating meson decays in a low scale left-right model, Phys. Rev. D 96, 035023 (2017).

[63] S. Mandal and N. Sinha, Favored $B_{c}$ decay modes to search for a Majorana neutrino, Phys. Rev. D 94, 033001 (2016).

[64] G. Cvetic and C. S. Kim, Rare decays of $B$ mesons via onshell sterile neutrinos, Phys. Rev. D 94, 053001 (2016); Erratum, Phys. Rev. D 95, 039901 (2017).

[65] D. Milanes, N. Quintero, and C. E. Vera, Sensitivity to Majorana neutrinos in $\Delta L=2$ decays of $B_{c}$ meson at LHCb, Phys. Rev. D 93, 094026 (2016).

[66] S. Antusch, E. Cazzato, and O. Fischer, Sterile neutrino searches via displaced vertices at LHCb, Phys. Lett. B 774, 114 (2017).

[67] K. Nakamura et al. (Particle Data Group Collaboration), Review of particle physics, J. Phys. G 37, 075021 (2010).

[68] FCC-ee Web page, http://tlep.web.cern.ch.

[69] D. Gorbunov and M. Shaposhnikov, How to find neutral leptons of the $\nu$ MSM?, J. High Energy Phys. 10 (2007) 015; Erratum, J. High Energy Phys. 11 (2013) 101.

[70] Na62 Web page, https://na62.web.cern.ch/na62/.

[71] T. Asaka and H. Ishida, Lepton number violation by heavy Majorana neutrino in $B$ decays, Phys. Lett. B 763, 393 (2016).

[72] G. J. Feldman and R. D. Cousins, A unified approach to the classical statistical analysis of small signals, Phys. Rev. D 57, 3873 (1998).

[73] R. Aaij et al. (LHCb Collaboration), Search for Majorana Neutrinos in $B^{-} \rightarrow \pi^{+} \mu^{-} \mu^{-}$Decays, Phys. Rev. Lett. 112, 131802 (2014).

[74] B. Shuve and M.E. Peskin, Revision of the LHCb limit on Majorana neutrinos, Phys. Rev. D 94, 113007 (2016).

[75] R. Aaij et al. (LHCb Collaboration), Measurement of the $b$-Quark Production Cross-Section in 7 and $13 \mathrm{TeV} p p$ Collisions, Phys. Rev. Lett. 118, 052002 (2017); Erratum, Phys. Rev. Lett. 119, 169901 (2017).

[76] R. Aaij et al. (LHCb Collaboration), Measurements of prompt charm production cross sections in $p p$ collisions at $\sqrt{s}=13 \mathrm{TeV}$, J. High Energy Phys. 03 (2016) 159; Erratum 05 (2017) 74.

[77] Our crude estimate is based on private communication with Vanya Belyaev from the LHCbcollaboration.

[78] R. Aaij et al. (LHCb Collaboration), Measurement of $B_{c}^{+}$ Production in Proton-Proton Collisions at $\sqrt{s}=8 \mathrm{TeV}$, Phys. Rev. Lett. 114, 132001 (2015).

[79] M. Artuso, in Proceedings of the ICHEP 2016 on behalf of LHCb collaboration, https://cds.cern.ch/record/2206834/ files/LHCb-TALK-2016-222.pdf.

[80] SHiP Web page, http://ship.web.cern.ch/ship/. 
[81] S. Alekhin et al., A facility to search for hidden particles at the CERN SPS: The SHiP physics case, Rep. Prog. Phys. 79, 124201 (2016).

[82] C. Ahdida et al. (SHiP Collaboration), Sensitivity of the SHiP experiment to heavy neutral leptons, J. High Energy Phys. 04 (2019) 077.

[83] D. Curtin et al., Long-lived particles at the energy frontier: The MATHUSLA physics case, Rep. Prog. Phys. 82, 116201 (2019).

[84] K. Bondarenko, A. Boyarsky, M. Ovchynnikov, and O. Ruchayskiy, Sensitivity of the intensity frontier experiments for neutrino and scalar portals: Analytic estimates, J. High Energy Phys. 08 (2019) 061.

[85] SuperKEKB Web page, http://www-superkekb.kek.jp.

[86] C. Patrignani et al. (Particle Data Group Collaboration), Review of particle physics, Chin. Phys. C 40, 100001 (2016).

[87] Our estimate is based on private communication with Karim Trabelsi from the Belle collaboration.

[88] D. Liventsev et al. (Belle Collaboration), Search for heavy neutrinos at Belle, Phys. Rev. D 87, 071102 (2013); Erratum, Phys. Rev. D 95, 099903 (2017).

[89] K. A. Olive et al. (Particle Data Group Collaboration), Review of particle physics, Chin. Phys. C 38, 090001 (2014).

[90] Y. Amhis et al. (Heavy Flavor Averaging Group (HFAG) Collaboration), Averages of $b$-hadron, $c$-hadron, and $\tau$-lepton properties as of summer 2014, arXiv:1412.7515.

[91] M. Drewes, The phenomenology of right handed neutrinos, Int. J. Mod. Phys. E 22, 1330019 (2013).

[92] F. F. Deppisch, P. S. Bhupal Dev, and A. Pilaftsis, Neutrinos and collider physics, New J. Phys. 17, 075019 (2015).

[93] A. Das, Searching for the minimal seesaw models at the LHC and beyond, Adv. High Energy Phys. 2018, 9785318 (2018).

[94] A. Caputo, P. Hernandez, M. Kekic, J. López-Pavón, and J. Salvado, The seesaw path to leptonic $C P$ violation, Eur. Phys. J. C 77, 258 (2017).

[95] G. Cvetič, A. Das, and J. Zamora-Saá, Probing heavy neutrino oscillations in rare $W$ boson decays, J. Phys. G 46, 075002 (2019).

[96] S. K. Kang, Roles of sterile neutrinos in particle physics and cosmology, Int. J. Mod. Phys. A 34, 1930005 (2019).

[97] G. Cvetič, A. Das, S. Tapia, and J. Zamora-Saá, Measuring the heavy neutrino oscillations in rare $\mathrm{W}$ boson decays at the Large Hadron Collider, arXiv:1905.03097 [J. Phys. G (to be published)].

[98] S. Tapia and J. Zamora-Saá, Exploring CP-violating heavy neutrino oscillations in rare tau decays at Belle II, arXiv: 1906.09470.

[99] K. Bondarenko, A. Boyarsky, D. Gorbunov, and O. Ruchayskiy, Phenomenology of GeV-scale heavy neutral leptons, J. High Energy Phys. 11 (2018) 032.

[100] R. W. Rasmussen and W. Winter, Perspectives for tests of neutrino mass generation at the $\mathrm{GeV}$ scale: Experimental reach versus theoretical predictions, Phys. Rev. D 94, 073004 (2016).
[101] A. Das, P. S. Bhupal Dev, and N. Okada, Direct bounds on electroweak scale pseudo-Dirac neutrinos from $\sqrt{\mathrm{s}}=$ 8 TeV LHC data, Phys. Lett. B 735, 364 (2014).

[102] P. S. B. Dev, A. Pilaftsis, and U.-K. Yang, New Production Mechanism for Heavy Neutrinos at the LHC, Phys. Rev. Lett. 112, 081801 (2014).

[103] A. Das, P. S. B. Dev, and C. S. Kim, Constraining sterile neutrinos from precision Higgs data, Phys. Rev. D 95, 115013 (2017).

[104] A. Das, Y. Gao, and T. Kamon, Heavy neutrino search via semileptonic Higgs decay at the LHC, Eur. Phys. J. C 79, 424 (2019).

[105] P. S. Bhupal Dev, R. Franceschini, and R. N. Mohapatra, Bounds on $\mathrm{TeV}$ seesaw models from LHC Higgs data, Phys. Rev. D 86, 093010 (2012).

[106] A. de Gouvea, W.-C. Huang, and J. Jenkins, Pseudo-Dirac neutrinos in the new standard model, Phys. Rev. D 80, 073007 (2009).

[107] A. de Gouvea, See-saw energy scale and the LSND anomaly, Phys. Rev. D 72, 033005 (2005).

[108] M. Cirelli, G. Marandella, A. Strumia, and F. Vissani, Probing oscillations into sterile neutrinos with cosmology, astrophysics and experiments, Nucl. Phys. B708, 215 (2005).

[109] A. Boyarsky, O. Ruchayskiy, and M. Shaposhnikov, The role of sterile neutrinos in cosmology and astrophysics, Annu. Rev. Nucl. Part. Sci. 59, 191 (2009).

[110] O. Ruchayskiy and A. Ivashko, Restrictions on the lifetime of sterile neutrinos from primordial nucleosynthesis, J. Cosmol. Astropart. Phys. 10 (2012) 014.

[111] F. Bergsma et al. (CHARM Collaboration), A search for decays of heavy neutrinos in the mass range $0.5-\mathrm{GeV}$ to 2.8-GeV, Phys. Lett. 166B, 473 (1986).

[112] P. Vilain et al. (CHARM II Collaboration), Search for heavy isosinglet neutrinos, Phys. Lett. B 343, 453 (1995); 351, 387 (1995).

[113] J. Orloff, A. N. Rozanov, and C. Santoni, Limits on the mixing of tau neutrino to heavy neutrinos, Phys. Lett. B 550, 8 (2002).

[114] P. Abreu et al. (DELPHI Collaboration), Search for neutral heavy leptons produced in $\mathrm{Z}$ decays, Z. Phys. C 74, 57 (1997); Erratum, Z. Phys. C 75, 580 (1997).

[115] A. Blondel, E. Graverini, N. Serra, and M. Shaposhnikov (FCC-ee Study Team Collaboration), Search for heavy right handed neutrinos at the FCC-ee, Nucl. Part. Phys. Proc. 273-275, 1883 (2016).

[116] A. Abada, V. De Romeri, S. Monteil, J. Orloff, and A. M. Teixeira, Indirect searches for sterile neutrinos at a highluminosity Z-factory, J. High Energy Phys. 04 (2015) 051.

[117] C. Adams et al. (LBNE Collaboration), The long-baseline neutrino experiment: Exploring fundamental symmetries of the universe, in Snowmass 2013: Workshop on Energy Frontier Seattle, USA, 2013, http://lss.fnal.gov/archive/ 2014/pub/fermilab-pub-14-022.pdf.

[118] G. Bernardi et al., Further limits on heavy neutrino couplings, Phys. Lett. B 203, 332 (1988).

[119] S. A. Baranov et al., Search for heavy neutrinos at the IHEP-JINR neutrino detector, Phys. Lett. B 302, 336 (1993). 
[120] M. Agostini et al. (GERDA Collaboration), Results on Neutrinoless Double- $\beta$ Decay of ${ }^{76} \mathrm{Ge}$ from Phase I of the GERDA Experiment, Phys. Rev. Lett. 111, 122503 (2013).

[121] A. V. Artamonov et al. (E949 Collaboration), Search for heavy neutrinos in $K^{+} \rightarrow \mu^{+} \nu_{H}$ decays, Phys. Rev. D 91, 052001 (2015); Erratum, Phys. Rev. D 91, 059903 (2015).

[122] A. Vaitaitis et al. (NuTeV, E815 Collaborations), Search for Neutral Heavy Leptons in a High-Energy Neutrino Beam, Phys. Rev. Lett. 83, 4943 (1999).

[123] S. Dube, D. Gadkari, and A. M. Thalapillil, Lepton-jets and low-mass sterile neutrinos at hadron colliders, Phys. Rev. D 96, 055031 (2017).

[124] F. del Aguila, J. de Blas, and M. Perez-Victoria, Effects of new leptons in electroweak precision data, Phys. Rev. D 78, 013010 (2008).

[125] E. Akhmedov, A. Kartavtsev, M. Lindner, L. Michaels, and J. Smirnov, Improving electro-weak fits with TeV-scale sterile neutrinos, J. High Energy Phys. 05 (2013) 081.

[126] J. de Blas, Electroweak limits on physics beyond the Standard Model, EPJ Web Conf. 60, 19008 (2013).

[127] C. O. Dib, J. C. Helo, M. Nayak, N. A. Neill, A. Soffer, and J. Zamora-Saa, Searching for a sterile neutrino in tau decays at $B$-factories, arXiv:1908.09719.

[128] E. Cortina Gil et al. (NA62 Collaboration), The beam and detector of the NA62 experiment at CERN, J. Instrum. 12, P05025 (2017).

[129] E. Cortina Gil et al. (NA62 Collaboration), Search for heavy neutral lepton production in $K^{+}$decays, Phys. Lett. B 778, 137 (2018).

[130] G. Lanfranchi (NA62 Collaboration), Search for hidden sector particles at NA62, Proc. Sci., EPS-HEP2017 (2017) 301.

[131] M. Drewes, J. Hajer, J. Klaric, and G. Lanfranchi, NA62 sensitivity to heavy neutral leptons in the low scale seesaw model, J. High Energy Phys. 07 (2018) 105.
[132] R. E. Shrock, General theory of weak leptonic and semileptonic decays. 1. Leptonic pseudoscalar meson decays, with associated tests for, and bounds on, neutrino masses and lepton mixing, Phys. Rev. D 24, 1232 (1981).

[133] R. E. Shrock, General theory of weak processes involving neutrinos. 2. Pure leptonic decays, Phys. Rev. D 24, 1275 (1981).

[134] M. Drewes, B. Garbrecht, D. Gueter, and J. Klaric, Testing the low scale seesaw and leptogenesis, J. High Energy Phys., 08 (2017) 018.

[135] F. Kling and S. Trojanowski, Heavy neutral leptons at FASER, Phys. Rev. D 97, 095016 (2018).

[136] G. Aad et al. (ATLAS Collaboration), Search for heavy neutral leptons in decays of $W$ bosons produced in $13 \mathrm{TeV}$ $p p$ collisions using prompt and displaced signatures with the ATLAS detector, arXiv:1905.09787.

[137] M. G. Aartsen et al. (IceCube Collaboration), Search for astrophysical tau neutrinos in three years of IceCube data, Phys. Rev. D 93, 022001 (2016).

[138] M. G. Aartsen et al. (IceCube Collaboration), The IceCube neutrino observatory: Instrumentation and online systems, J. Instrum. 12, P03012 (2017).

[139] M. G. Aartsen et al. (IceCube Collaboration), Measurement of Atmospheric Neutrino Oscillations at 6-56 GeV with IceCube DeepCore, Phys. Rev. Lett. 120, 071801 (2018).

[140] P. Coloma, P. A. N. Machado, I. Martinez-Soler, and I. M. Shoemaker, Double-Cascade Events from New Physics in Icecube, Phys. Rev. Lett. 119, 201804 (2017).

[141] R. Alonso, M. Dhen, M. B. Gavela, and T. Hambye, Muon conversion to electron in nuclei in type-I seesaw models, J. High Energy Phys. 01 (2013) 118.

[142] B. Aubert et al. (BABAR Collaboration), Search for Lepton-Flavor and Lepton-Number Violation in the Decay $\tau^{-} \rightarrow \ell^{\mp} h^{ \pm} h^{\prime-}$, Phys. Rev. Lett. 95, 191801 (2005).

[143] J. Zamora-Saa, Resonant $C P$ violation in rare $\tau^{ \pm}$decays, J. High Energy Phys. 05 (2017) 110. 